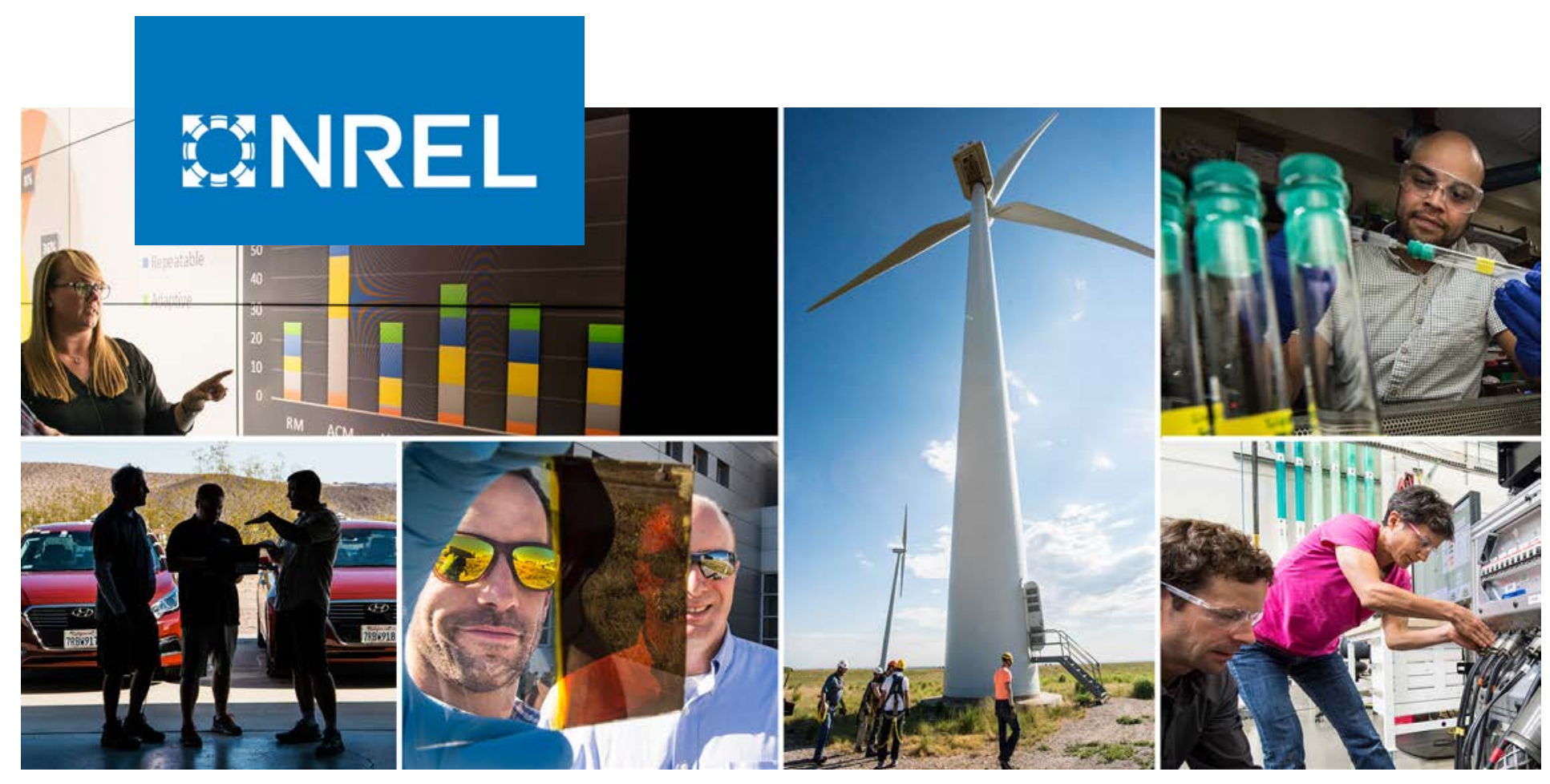

\title{
Aeroacoustics Noise Model of OpenFAST
}

Pietro Bortolotti, ${ }^{1}$ Emmanuel Branlard, ${ }^{1}$ Andy Platt, ${ }^{1}$

Patrick J. Moriarty, ${ }^{1}$ Carlo Sucameli, ${ }^{2}$ and

Carlo L. Bottasso ${ }^{2}$

${ }^{1}$ National Renewable Energy Laboratory

${ }^{2}$ Technical University of Munich, Germany

NREL is a national laboratory of the U.S. Department of Energy

Office of Energy Efficiency \& Renewable Energy

Operated by the Alliance for Sustainable Energy, LLC

This report is available at no cost from the National Renewable Energy Laboratory (NREL) at www.nrel.gov/publications.
Technical Report

NREL/TP-5000-75731

August 2020 


\title{
GNREL
}

\section{Aeroacoustics Noise Model of OpenFAST}

\author{
Pietro Bortolotti, ${ }^{1}$ Emmanuel Branlard, ${ }^{1}$ Andy Platt, ${ }^{1}$ \\ Patrick J. Moriarty, ${ }^{1}$ Carlo Sucameli, ${ }^{2}$ and \\ Carlo L. Bottasso ${ }^{2}$
}

${ }^{1}$ National Renewable Energy Laboratory

${ }^{2}$ Technical University of Munich, Germany

\author{
Suggested Citation \\ Bortolotti, Pietro, Emmanuel Branlard, Andy Platt, Patrick J. Moriarty, Carlo Sucameli, and \\ Carlo L. Bottasso. 2020. Aeroacoustics Noise Model of OpenFAST. Golden, CO: National \\ Renewable Energy Laboratory. NREL/TP-5000-75731. \\ https://www.nrel.gov/docs/fy20osti/75731.pdf.
}

NREL is a national laboratory of the U.S. Department of Energy Office of Energy Efficiency \& Renewable Energy Operated by the Alliance for Sustainable Energy, LLC

This report is available at no cost from the National Renewable Energy Laboratory (NREL) at www.nrel.gov/publications.

Contract No. DE-AC36-08GO28308
Technical Report NREL/TP-5000-75731 August 2020

National Renewable Energy Laboratory 15013 Denver West Parkway Golden, CO 80401 303-275-3000 • www.nrel.gov 


\section{NOTICE}

This work was authored in part by the National Renewable Energy Laboratory, operated by Alliance for Sustainable Energy, LLC, for the U.S. Department of Energy (DOE) under Contract No. DE-AC36-08GO28308. Funding provided by the U.S. Department of Energy Office of Energy Efficiency and Renewable Energy Wind Energy Technologies Office. The views expressed herein do not necessarily represent the views of the DOE or the U.S. Government.

This report is available at no cost from the National Renewable Energy Laboratory (NREL) at www.nrel.gov/publications.

U.S. Department of Energy (DOE) reports produced after 1991 and a growing number of pre-1991 documents are available free via www.OSTI.gov.

Cover Photos by Dennis Schroeder: (clockwise, left to right) NREL 51934, NREL 45897, NREL 42160, NREL 45891, NREL 48097, NREL 46526.

NREL prints on paper that contains recycled content. 


\section{Acknowledgments}

The National Renewable Energy Laboratory (NREL) acknowledges the U.S. Department of Energy Wind Energy Technologies Office for supporting this work under the Office of Energy Efficiency and Renewable Energy's technology research and development activities. Authors thank Emre Barlas and Matthew Phillips for working on previous versions of the code. The guidance and support of Nick Johnson and Nicholas Hamilton, project leaders at NREL, are also acknowledged. Finally, the reviews of Jason Jonkman (NREL) and Franck Bertagnolio (Denmark Technical University) greatly improved this work. 


\section{List of Acronyms}

$\begin{array}{ll}\text { BPM } & \text { Brooks-Pope-Marcolini airfoil noise model } \\ \text { dB } & \text { decibels } \\ \text { dBA } & \text { A-weighted decibels } \\ \text { deg } & \text { degrees } \\ \mathrm{Hz} & \text { hertz } \\ \mathrm{IEA} & \text { International Energy Agency } \\ \mathrm{kg} & \text { kilograms } \\ \mathrm{kHz} & \text { kilohertz } \\ \mathrm{LFC} & \text { low-frequency correction } \\ \mathrm{m} & \text { meters } \\ \mathrm{N} & \text { newtons } \\ \mathrm{NREL} & \text { National Renewable Energy Laboratory } \\ \text { rad } & \text { radians } \\ \mathrm{s} & \text { seconds } \\ \text { SPL } & \text { sound pressure level } \\ \text { TBL } & \text { turbulent boundary layer } \\ \text { TBL-TE } & \text { turbulent boundary layer }- \text { trailing edge } \\ \text { TNO } & \text { a Netherlands organization for applied scientific research } \\ \text { TE } & \text { trailing edge } \\ \text { TI } & \text { turbulent inflow } \\ \text { TUM } & \text { Technical University of Munich }\end{array}$




\section{List of Symbols}

\begin{tabular}{|c|c|c|}
\hline$l$ & low frequency & \\
\hline$h$ & high frequency & \\
\hline$p$ & airfoil pressure side & \\
\hline$s$ & airfoil suction side & \\
\hline$t$ & turbulence & \\
\hline 0 & reference & \\
\hline 1 & parallel to airfoil chord & \\
\hline 2 & normal to airfoil chord & \\
\hline 3 & blade spanwise direction & \\
\hline$\alpha$ & angle of attack & {$[\mathrm{rad}]$} \\
\hline$\beta^{2}$ & Prandtl-Glauert correction factor & {$[-]$} \\
\hline$\delta$ & airfoil boundary layer thickness & {$[-]$} \\
\hline$\delta^{*}$ & airfoil boundary layer displacement thickness & {$[-]$} \\
\hline$\theta$ & airfoil boundary layer momentum thickness & {$[-]$} \\
\hline$\Theta_{e}, \Phi_{e}$ & angles between emitter and observer & {$[\mathrm{rad}]$} \\
\hline$\rho$ & air density & {$\left[\mathrm{kg} / \mathrm{m}^{3}\right]$} \\
\hline$\omega$ & radial frequency & {$[\mathrm{rad} / \mathrm{s}]$} \\
\hline$A_{w}$ & A-weight & {$[\mathrm{dB}]$} \\
\hline$c$ & speed of sound & {$[\mathrm{m} / \mathrm{s}]$} \\
\hline$c_{i}$ & chord at blade spanwise position $\mathrm{i}$ & {$[\mathrm{m}]$} \\
\hline$d$ & blade span at station $\mathrm{i}$ & {$[\mathrm{m}]$} \\
\hline $\bar{D}$ & directivity function & {$[-]$} \\
\hline$f$ & frequency & {$[\mathrm{Hz}]$} \\
\hline$G$ & empirical function & {$[-]$} \\
\hline$h$ & height of the trailing edge thickness & {$[\mathrm{m}]$} \\
\hline$H$ & airfoil kinematic shape factor & {$[-]$} \\
\hline$I$ & turbulence intensity & {$[-]$} \\
\hline$k$ & wave number & {$\left[\mathrm{m}^{-1}\right]$} \\
\hline $\bar{k}, \hat{k}$ & nondimensional wave number & {$[-]$} \\
\hline$\Delta K_{1}, K_{1}, K_{2}$ & empirical parameters of the BPM model & {$[-]$} \\
\hline$l$ & spanwise extent of the separation zone from blade tip & {$[\mathrm{m}]$} \\
\hline$L$ & lift force & {$[\mathrm{N}]$} \\
\hline$L_{t}$ & length scale & {$[\mathrm{m}]$} \\
\hline$\dot{M}$ & Mach number & {$[-]$} \\
\hline$M_{c}$ & Mach number past the trailing edge & {$[-]$} \\
\hline$r_{e}$ & effective observer distance & {$[\mathrm{m}]$} \\
\hline$R e$ & Reynolds number & {$[-]$} \\
\hline$S^{2}$ & Sears function & {$[-]$} \\
\hline$S t$ & Strouhal number & {$[-]$} \\
\hline$t_{x}$ & relative thickness of the airfoil at chordwise position $\mathrm{x}$ & {$[-]$} \\
\hline$U$ & local inflow velocity & {$[\mathrm{m} / \mathrm{s}]$} \\
\hline$y$ & blade spanwise position & {$[\mathrm{m}]$} \\
\hline$z$ & height above the ground & {$[\mathrm{m}]$} \\
\hline$z_{0}$ & ground surface roughness & {$[\mathrm{m}]$} \\
\hline
\end{tabular}




\section{Executive Summary}

This report describes theory and application of a newly released model of OpenFAST to simulate the aeroacoustics noise generated by the rotor of an arbitrary wind turbine. OpenFAST is a fully open-source, publicly available wind turbine analysis tool actively developed at the National Renewable Energy Laboratory. The aeroacoustics model, which is also fully open source and publicly available, is based on work performed over the past three decades. Frequency-based models for turbulent inflow; turbulent boundary layer - trailing edge; laminar boundary layer vortex shedding; tip vortex; and trailing edge bluntness - vortex shedding noise mechanisms are included. A simple directivity model is also included. The appendices of this report describe in detail the inputs to the model and how to find them in the input files of OpenFAST. A live version of this documentation is available at https://openfast.readthedocs.io/.

The noise models are exercised simulating the aeroacoustics noise emissions of the International Energy Agency Wind Task 37 land-based reference wind turbine. A code-to-code comparison between the implementation presented here and the implementation available at the Wind Energy Institute of the Technical University of Munich, Germany, is also presented. 


\section{Table of Contents}

Acknowledgments ......................................................................................................................... iii

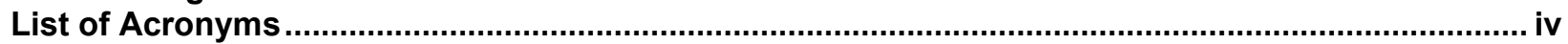

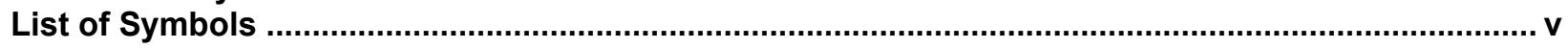

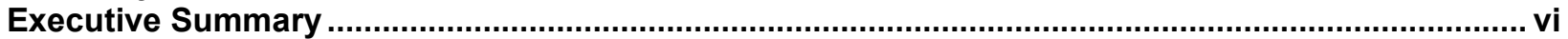

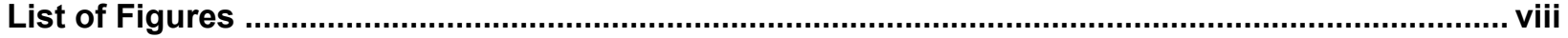

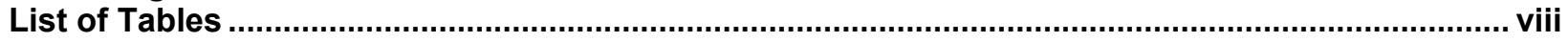

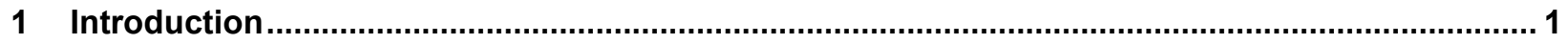

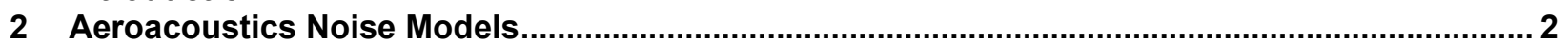

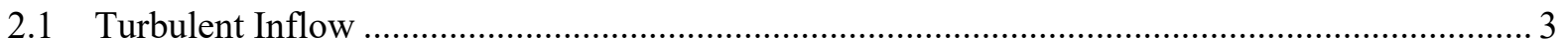

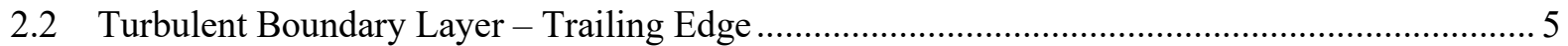

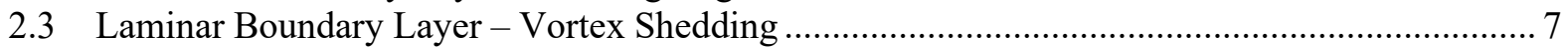

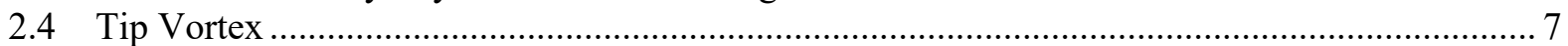

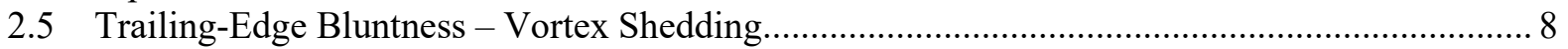

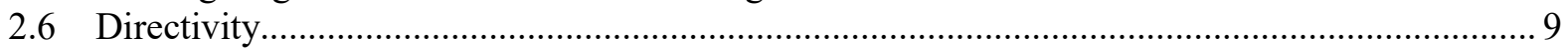

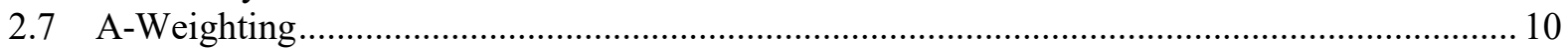

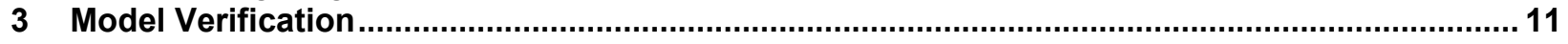

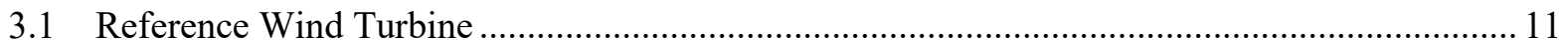

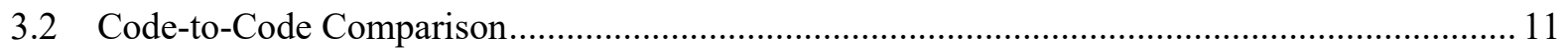

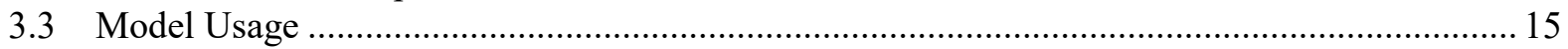

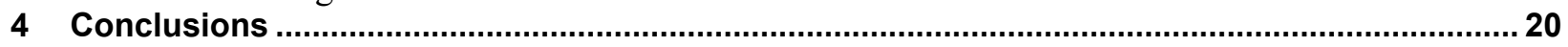

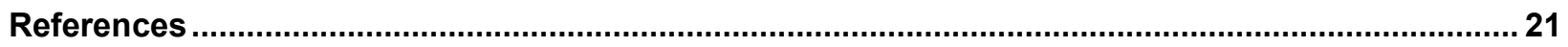

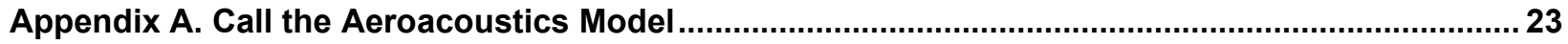

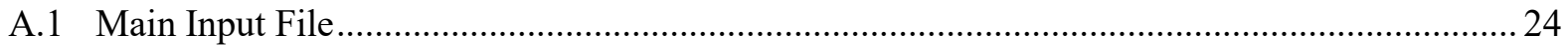

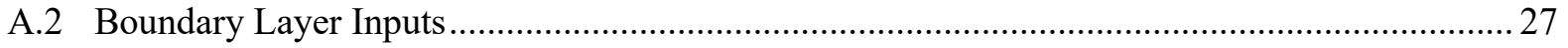

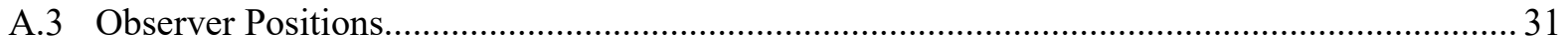

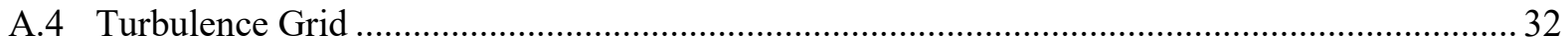

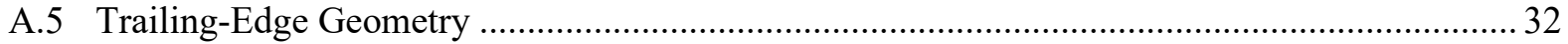




\section{List of Figures}

Figure 1. Angles used in the directivity function (Brooks et al. 1989; Moriarty and Migliore 2003)...9

Figure 2. Code-to-code comparison for the turbulent inflow models ........................................... 12

Figure 3. Code-to-code comparison for the BPM and TNO TBL-TE models. The boundary layer properties are estimated from either the BPM model (BPM BL) or defined by the user (Ext BL)

Figure 4. Location and numbering of the observers ................................................................... 14

Figure 5. Comparison of overall sound pressure levels for the observers shown in Figure 4 ........ 14

Figure 6. Map of the overall SPL of the reference wind turbine at a 2-m height from Simplified Guidati and BPM TBL-TE noise models. The wind turbine is located at $x=0, y=0$. A steady wind

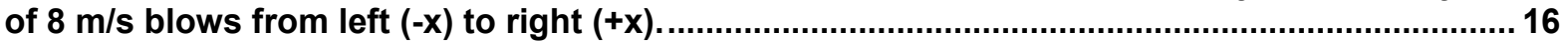

Figure 7. Comparison between absolute and A-weighted SPL ..................................................... 17

Figure 8. Nonweighted SPL spectra of the various noise mechanisms ....................................... 18

Figure 9. Map of the overall SPL of the rotor of the reference wind turbine from the Simplified Guidati and BPM TBL-TE noise models. The observer is located $175 \mathrm{~m}$ downwind at a height of $2 \mathrm{~m}$.

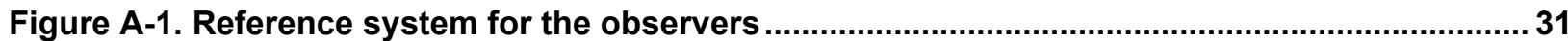

Figure A-2. Geometric parameters $\Psi$ and $\mathrm{h}$ of the trailing-edge bluntness ..................................... 32

\section{List of Tables}

Table 1. Main Characteristics of the IEA Wind Task 37 Land-Based Reference Wind Turbine 


\section{Introduction}

The increasing penetration of wind energy into the electricity mix has been possible thanks to a constantly growing installed capacity, which has so far been mostly located on land. Land-based installations are, however, increasingly constrained by local ordinances and an often-limiting factor that comprises maximum allowable levels of noise. To further increase the number of land-based installations, it is important to develop accurate modeling tools to estimate the noise generated by wind turbines. This allows for a more accurate assessment of the noise emissions and the possibility to design quieter wind turbines.

Wind turbines emit two main sources of noise:

- Aeroacoustics noise from the interaction between rotor blades and the turbulent atmospheric boundary layer

- Mechanical noise from the nacelle component, mostly the gearbox, generator, and yaw mechanism.

This work targets the first class of noise generation and aims at providing a set of open-source models to estimate the aeroacoustics noise generated by an arbitrary wind turbine rotor. The models are implemented in Fortran and are fully coupled to the aeroservoelastic wind turbine simulator OpenFAST. The code is available in the GitHub repository of OpenFAST. ${ }^{1}$ The code builds on the implementation of NAFNoise and the documentation presented in Moriarty and Migliore (2003) and Moriarty (2005). OpenFAST is implemented as a modularization framework and the aeroacoustics model is implemented as a submodule of AeroDyn (Moriarty and Hansen 2005).

The set of models is described in Section 2 and exercised on the noise estimate of the International Energy Agency (IEA) land-based reference wind turbine in Section 3. In Section 3, we also show a comparison to results obtained running the noise models implemented at the Technical University of Munich. The report closes with conclusions, an outlook on future work, and appendices, where the input files to OpenFAST are presented.

\footnotetext{
${ }^{1}$ https://github.com/OpenFAST/openfast
} 


\section{Aeroacoustics Noise Models}

The aeroacoustics noise of wind turbine rotors emanates from pressure oscillations that are generated along the blades and propagate in the atmosphere. This source of noise has been historically simulated with models characterized by different fidelity levels. At lower fidelity, models correlated aeroacoustics noise with rotor thrust and torque (Lowson 1970; Viterna 1981). At higher fidelity, three-dimensional incompressible computational fluid dynamics models are coupled with the Ffowcs Williams-Hawkings model to propagate pressure oscillations generated along the surface of the rotor blades to the far field (Klein et al. 2018). The latter models are often only suitable to estimate noise at low frequency because capturing noise in the audible range, which is commonly defined between 20 (hertz) $\mathrm{Hz}$ and 20 kilohertz (kHz), requires a very fine space-time discretization with enormous computational costs.

For the audible range, a variety of models is available in the public domain, and Sucameli et al. (2018) offers the most recent literature review. These models have inputs that match the inputs and outputs of modern aeroservoelastic solvers, such as OpenFAST, and have therefore often been coupled together. Further, the computational costs of these acoustic models are similar to the costs of modern aeroservoelastic solvers, which has facilitated the coupling.

Models have targeted different noise generation mechanisms following the distinction defined by Brooks, Pope, and Marcolini (BPM) (1989), and the mechanism of turbulent inflow noise. The latter represents a broadband noise source that is generated when a body of arbitrary shape experiences an unsteady lift because of the presence of an incident turbulent flow. For an airfoil, this phenomenon can be interpreted as leading-edge noise. Turbulent inflow noise was the topic of multiple investigations over the past decades and, as a result, multiple models have been published (Sucameli et al. 2018). The BPM model includes five mechanisms of noise generation for an airfoil immersed in a flow:

1. Turbulent boundary layer - trailing edge (TBL-TE)

2. Separation stall

3. Laminar boundary layer - vortex shedding

4. Tip vortex

5. Trailing-edge bluntness - vortex shedding.

For the five mechanisms, semiempirical models were initially defined for the NACA 0012 airfoil. The BPM model is still a popular model for wind turbine noise prediction, and subsequent studies have improved the model by removing some of the assumptions originally adopted. Recent studies have especially focused on the TBL-TE mechanism, which is commonly the dominant noise source of modern wind turbines. As a result, each noise source defined in the BPM model now has a variety of permutations.

The following subsections describe the details of each mechanism and the models implemented in this model of OpenFAST. 


\subsection{Turbulent Inflow}

A body of any arbitrary shape, when immersed in a turbulent flow, generates surface pressure fluctuations. Over the years, several formulations of the turbulent inflow noise model have been developed (Sucameli et al. 2018). In this model of OpenFAST, the formulation defined in Moriarty et al. (2004) is adopted. The formulation is based on the model of Amiet (Amiet 1975; Amiet and Paterson 1976) and is presented in Section 2.1.1. Additionally, the user can activate the correction defined by Moriarty et al. (2005), which builds upon the Amiet model and accounts for the thickness of the airfoils adopted along the blade span. This second model is named Simplified Guidati and is presented in Section 2.1.2.

\subsubsection{Amiet model}

The formulation is based on work from Amiet (1975) and Amiet and Paterson (1976), and it represents the blade as a flat plate and neglects the shape of the airfoil.

The model starts by first computing the wave number, $k_{1}$, for a given frequency $f$ :

$$
k_{1}=\frac{2 \pi f}{U_{1}}
$$

where $U_{1}$ is the incident inflow velocity on the profile. From $k_{1}$, the wave numbers $\bar{k}_{1}$ and $\hat{k}_{1}$ are computed:

$$
\begin{aligned}
& \bar{k}_{1}=\frac{k_{1} c_{i}}{2} \\
& \hat{k}_{1}=\frac{k_{1}}{k_{e}}
\end{aligned}
$$

where $c_{i}$ is the local chord, and $k_{e}$ is the wave number range of energy containing eddies, defined as:

$$
k_{e}=\frac{3}{4 L_{t}} .
$$

$\mathrm{L}_{\mathrm{t}}$ is the turbulent length scale, and many different formulations have been proposed over the years. As default implementation, $L_{t}$ is defined following the formulation proposed in Zhu et al. (2005):

$$
L_{t}=25 z^{0.35} z_{0}^{-0.063}
$$

where $z$ is the height above the ground of the leading edge of section $i$ at a given instant, $t$, while $z_{0}$ is the surface roughness. Note that setting $L_{t}$ appropriately is a challenge, and advanced users of this model may want to validate this formulation against experimental data.

The value of sound pressure level (SPL) is expressed in one-third octave bands at the given frequency, $f$, originated at the given blade station, $i$, which can be computed as: 


$$
S P L_{T I}=10 \log _{10}\left(\rho^{2} c^{4} \frac{L_{t} d}{2 r_{e}^{2}} M^{5} I_{1}^{2} \frac{\hat{k}_{1}^{3}}{\left(1+\hat{k}_{1}^{2}\right)^{\frac{7}{3}}} \bar{D}\right)+78.4
$$

where $\rho$ is the air density, $c$ the speed of sound, $d$ the blade element span, $r_{e}$ the effective distance between leading edge and observer, $M$ the Mach number, $I_{1}$ the turbulence intensity of the airfoil inflow, and $\bar{D}$ the directivity term. $\bar{D}$ is different below $\left(\bar{D}_{l}\right)$ and above $\left(\bar{D}_{h}\right)$ a certain frequency, which is named "cut-off" and defined as:

$$
f_{c o}=\frac{10 U_{1}}{\pi c_{i}}
$$

The formulations of $\bar{D}_{h}$ and $\bar{D}_{l}$ are presented in Section 2.6.

The current implementation offers two approaches to estimate $I_{1}$. The first one is through a userdefined grid of $I_{1}$; see Appendix A.4. The second option is to have the code reconstructing $I_{1}$ from the turbulent wind grid, where the code computes the airfoil relative position of each blade section, $i$, at every time instant and, given the rotor speed, reconstructs the inflow component, $I_{1}$, of the turbulence intensity.

Two corrections to this model are also implemented. The first one comprises a correction for the angle of attack, $\alpha$, in which the effect is neglected in the original formulation from Amiet (1975) and Amiet and Peterson (1976). This correction is formulated as:

$$
S P L_{T I}=S P L_{T I}+10 \log _{10}\left(1+9 a^{2}\right) .
$$

The second correction is called low-frequency correction $(L F C)$, and is formulated as:

$$
\begin{aligned}
& S^{2}=\left(\frac{2 \pi \bar{k}_{1}}{\beta^{2}}+\left(1+2.4 \frac{\bar{k}_{1}}{\beta^{2}}\right)^{-1}\right)^{-1} \\
& L F C=10 S^{2} M \bar{k}_{1}^{2} \beta^{-2} \\
& S P L_{T I}=S P L_{T I}+10 \log _{10}\left(\frac{L F C}{1+L F C}\right) .
\end{aligned}
$$

In Eq. 9 and Eq. 10, $S^{2}$ represents the squared Sears function, and $\beta^{2}$ is the Prandtl-Glauert correction factor, which is defined as:

$$
\beta^{2}=1-M^{2}
$$

It is worth stressing that numerous alternative formulations of the turbulent inflow noise model exist (Sucameli et al. 2018), where the main differences comprise different definitions of $L_{t}$ and $k_{1}$. 


\subsubsection{Simplified Guidati}

Sound spectra are often overpredicted by the Amiet model implemented here. Guidati et al. (1997) derived a correction to the sound pressure levels by adding a term considering shape and camber of the airfoil profiles, but the method proved computationally too expensive for wind turbine simulations. Moriarty et al. (2005) proposed a simplified model based on geometric characteristics of six wind turbine airfoils. The validity of the correction is limited to Mach numbers on the order of $0.1 \approx 0.2$ and Strouhal number $S t$ below 75. St is defined based on airfoil chord and mean inflow velocity:

$$
S t=\frac{f c_{i}}{U_{1}} .
$$

The formula for the correction to the noise spectra is provided in Eq. 4 in Moriarty et al. (2005):

$$
\begin{aligned}
& t=t_{1 \%}+t_{10 \%} \\
& \Delta S P L_{T I}=-\left(1.123 t+5.317 t^{2}\right)(2 \pi S t+5)
\end{aligned}
$$

where $t_{x \%}$ is the relative thickness of the profile at $x$ position along the chord (i.e., $0 \%$ being the leading edge and $100 \%$ the trailing edge).

It should be highlighted here that a validation campaign was conducted in a wind tunnel on twodimensional airfoils (Moriarty et al. 2004), returning a fairly poor match between the Simplified Guidati model and the experimental results. Therefore, a correction of +10 decibels $(\mathrm{dB})$ on the SPL levels across the whole frequency spectrum was proposed. This correction is still implemented, but a validation at turbine level should assess the accuracy of the models for turbulent inflow. It should also be noted that the code currently does not check whether Mach and Strouhal numbers are within the range of validity of this model.

\subsection{Turbulent Boundary Layer - Trailing Edge}

Airfoils immersed in a flow develop a boundary layer, which at high Reynolds numbers is turbulent. When the turbulence passes over the trailing edge, noise is generated. This noise source was named TBL-TE in Brooks et al. (1989) and it is a relevant source of aeroacoustics noise for modern wind turbine rotors. Two formulations of TBL-TE noise are implemented in the code: (1) the original formulation from the BPM model, described in Section 2.2.1, and (2) a more recent model developed at the Dutch research institute, TNO, described in Section 2.2.2. Both models take as input the characteristics of the airfoil boundary layer. These must be provided by the user and are discussed in Appendix A.2.

\subsubsection{BPM}

The $S P L$ of the TBL-TE noise in the BPM model is made from three contributions:

$$
S P L_{T B L-T E}=10 \log _{10}\left(10^{\frac{S P L_{p}}{10}}+10^{\frac{S P L_{S}}{10}}+10^{\frac{S P L_{\alpha}}{10}}\right)
$$


where the subscripts $\mathrm{p}, \mathrm{s}$, and $\alpha$ refer to the contributions of pressure side, suction side, and angle of attack, respectively. The equations describing the three contributions are described in great detail in Section 5.1.2, in Brooks et al. (1989), and are summarized here.

For the suction and pressure contributions, the equations are:

$$
\begin{aligned}
& S P L_{p}=10 \log _{10}\left(\frac{\delta_{p}^{*} M^{5} d \bar{D}_{h}}{r_{e}^{2}}\right)+A\left(\frac{S t_{p}}{S t_{1}}\right)+\left(K_{1}-3\right)+\Delta K_{1} \\
& S P L_{s}=10 \log _{10}\left(\frac{\delta_{s}^{*} M^{5} d \bar{D}_{h}}{r_{e}^{2}}\right)+A\left(\frac{S t_{s}}{S t_{1}}\right)+\left(K_{1}-3\right) .
\end{aligned}
$$

The terms in the equations, which are also described in the nomenclature at the beginning of this document, list $\delta^{*}$ as the boundary layer displacement thickness on either side of the airfoil, $S t$ as the Strouhal number based on $\delta^{*}$, and $A, A^{\prime}, B, \Delta K_{1}, K_{1}$, and $K_{2}$ as empirical functions based on St.

For the angle-of-attack contribution, a distinction is made above and below the stall angle, which in the original BPM model is set equal to 12.5 degrees, whereas it is here assumed to be the actual stall angle of attack of the airfoil at blade station i. Below stall, $S P L_{\alpha}$ is equal to:

$$
S P L_{\alpha}=10 \log _{10}\left(\frac{\delta_{s}^{*} M^{5} d \bar{D}_{h}}{r_{e}^{2}}\right)+B\left(\frac{S t_{s}}{S t_{2}}\right)+K_{2} .
$$

At angles of attack above the stall point, the flow along the profile is fully separated and noise radiates from the whole chord. $S P L_{p}$ and $S P L_{S}$ are then set equal to $-\infty$, whereas $S P L_{\alpha}$ becomes:

$$
S P L_{\alpha}=10 \log _{10}\left(\frac{\delta_{s}^{*} M^{5} d \bar{D}_{l}}{r_{e}^{2}}\right)+A^{\prime}\left(\frac{S t_{s}}{S t_{2}}\right)+K_{2}
$$

Notably, above stall the low-frequency directivity $\bar{D}_{l}$ is adopted in Eqs. 18 and 19 (see Section 2.6).

\subsubsection{TNO model}

The TNO model is a more recent model to simulate the noise emitted by the vortices shed at the trailing edge of the blades and was formulated by Parchen (1998). The implementation adopted here is the one described in Moriarty et al. (2005). The TNO model uses the spectrum of the

wave number, $\bar{k}$, of unsteady surface pressures to estimate the far-field noise. The spectrum, $P$, is assumed to be:

$$
\begin{aligned}
P\left(k_{1}, k_{3}, \omega\right)= & 4 \rho_{0}^{2} \frac{k_{1}^{2}}{k_{1}^{2}+k_{3}^{2}} \int_{0}^{\delta} L_{2} \overline{u_{2}^{2}}\left(\frac{\partial U_{1}}{\partial x_{2}}\right)^{2} \phi_{22}\left(k_{1}, k_{3}, \omega\right) \phi_{m}(\omega \\
& \left.-U_{c}\left(x_{2}\right) k_{1}\right) e^{\left(-2|\bar{k}| x_{2}\right)} d x_{2} .
\end{aligned}
$$


In the equation, the indices 1,2 , and 3 refer to the directions parallel to the airfoil chord, normal to the airfoil chord, and along span, respectively; $\phi_{22}$ is the vertical velocity fluctuation spectrum; $\phi_{m}$ is the moving axis spectrum; and $U_{c}$ is the convection velocity of the eddies along the trailing edge. Lastly, $L_{2}$ is the vertical correlation length, perpendicular to the chord length, which indicates the vertical extension of the vortices that convect over the trailing edge. In this work, $L_{2}$ is assumed equal to the mixing length, $L_{m}$ (Moriarty et al. 2005). This decision is partially arbitrary, and dedicated research should better assess the correct integral length to be adopted within the TNO model.

From $P$, the far-field spectrum, $S(\omega)$, is computed as:

$$
S(\omega)=\frac{d \bar{D}_{h}}{4 \pi r_{e}^{2}} \int_{0}^{10 \frac{\omega}{M c}} \frac{\omega}{c k_{1}} P\left(k_{1}, 0, \omega\right) d k_{1}
$$

The implementation of the TNO model is identical to the one described in Moriarty et al. (2005). The inputs to the model are generated from the boundary layer characteristics provided by the user (see Appendix A.2).

\subsection{Laminar Boundary Layer - Vortex Shedding}

Another source of airfoil self-noise noise included in the BPM model is the noise generated by a feedback loop between vortices being shed at the trailing edge and instability waves in the laminar boundary layer. This noise is typically distributed on a narrow band of frequencies and occurs when the boundary layer of the airfoil remains laminar. This may occur in the inboard region of smaller wind turbines, where the Reynolds number can be smaller than 1 million, but hardly occurs in modern rotors that operate at a Reynolds number one order of magnitude larger. The formula to estimate the noise spectrum in a one-third-octave presentation is:

$$
S P L_{L B L-V S}=10 \log _{10}\left(\frac{\delta_{p} M^{5} d \bar{D}_{h}}{r_{e}^{2}}\right)+G_{1}\left(\frac{S t^{\prime}}{S t_{\text {peak }}^{\prime}}\right)+G_{2}\left[\frac{R e_{c}}{\left(R e_{c}\right)_{0}}\right]+G_{3}\left(\alpha_{*}\right)
$$

where $G$ represents empirical functions, $S t^{\prime}$ peak is the peak Strouhal number function of $R e_{c}$, which is the Reynolds number at chord, $c_{i}$. The subscript 0 refers to a reference Reynolds number that is a function of the angle of attack (Brooks et al. 1989).

\subsection{Tip Vortex}

The vortices generated at blade tips are another source of noise of the BPM model. Although rarely relevant in modern wind turbines, the possibility to include this noise source is offered. The sound pressure level is estimated as:

$$
S P L_{\text {Tip }}=10 \log _{10}\left(\frac{M^{2} M_{\text {max }}^{2} l^{2} \bar{D}_{h}}{r_{e}^{2}}\right)-30.5\left(\log _{10} S t^{\prime \prime}+0.3\right)^{2}+126
$$

where $M_{\max }=M_{\max }\left(\alpha_{\text {tip }}\right)$ is the maximum Mach number, measured near the blade tip within the separated flow region that is assumed to depend on $\alpha_{t i p}$, which is the angle of attack at the 
tip; $l$ is the spanwise extent of the separation zone; and $S t^{\prime \prime \prime}$ is the Strouhal number based on $l$. For a round shape of the tip, $l$ is estimated as:

$$
l=c_{i} 0.008 \alpha_{\text {tip }}
$$

where $\alpha_{\text {tip }}$ is the angle of attack of the tip region to the incoming flow. For a square tip, the BPM model estimates $l$ based on the quantity, $\alpha^{\prime}$ tip, which is defined as:

$$
\alpha_{t i p}^{\prime}=\left[\left(\frac{\frac{\partial L^{\prime}}{\partial y}}{\left(\frac{\partial L^{\prime}}{\partial y}\right)_{r e f}}\right)_{y \rightarrow t i p}\right] \alpha_{t i p}
$$

where $L^{\prime}$ is the lift per unit span along the blade at position $y$. For $\alpha^{\prime}{ }_{\text {tip }}$ between 0 and 2 degrees, $l$ becomes:

$$
l=c_{i}\left(0.0230+0.0169 \alpha^{\prime}{ }_{t i p}\right)
$$

while for $\alpha^{\prime}{ }_{t i p}$ larger than 2 degrees, $l$ is:

$$
l=c_{i}\left(0.0378+0.0095 \alpha^{\prime}{ }_{t i p}\right) \text {. }
$$

However, it must be noted that, unfortunately, $\alpha_{\text {tip }}$ is not a reliable output of standard aeroelastic models and the impossibility to accurately determine $\alpha_{\text {tip }}$ weakens the formulation of the tip vortex noise.

\subsection{Trailing-Edge Bluntness - Vortex Shedding}

Lastly, wind turbine blades are often characterized by a finite height of the trailing edge, which generates noise as a result of vortex shedding. The frequency and amplitude of this noise source depends on the geometry of the trailing edge and is typically characterized by a tonal nature. Adopting flatback and truncated airfoils far outboard along the blade may strengthen this noise source. When this noise source is activated, the user is asked to provide the distribution along the blade span of the blunt thickness of the trailing edge, $h$, and the solid angle between the suction and pressure sides of the airfoil, $\Psi$ (see Appendix A.5). $h$ and $\Psi$ are inputs to the equation:

$$
S P L_{T E B-V S}=10 \log _{10}\left(\frac{\delta_{p}^{*} M^{5} d \bar{D}_{h}}{r_{e}^{2}}\right)+G_{4}\left(\frac{h}{\delta_{\text {avg }}^{*}}, \Psi\right)+G_{5}\left(\frac{h}{\delta_{\text {avg }}^{*}}, \Psi, \frac{S t^{\prime \prime}}{S t^{\prime \prime} \text { peak }}\right) .
$$

In the equation, $\delta_{\text {avg }}^{*}$ is the average displacement thickness for both sides of the airfoil. Note that this noise source is very sensitive to $h$ and $\Psi$, which, therefore, should be estimated accurately. 


\subsection{Directivity}

The position of one or more observers is specified by the user, as described in Appendix A.3. The directivity from the BPM model is adopted in this implementation (Brooks et al. 1989). The directivity term, $\bar{D}$, corrects the $S P L$ depending on the relative position of the observer to the emitter. The position is described by the spanwise directivity angle, $\Phi_{e}$, and by the chordwise directivity angle, $\Theta_{e}$, which are schematically represented in Figure 1 and defined as:

$$
\begin{aligned}
& \Phi_{e}=\operatorname{atan}\left(\frac{z_{e}}{y_{e}}\right) \\
& \Theta_{e}=\operatorname{atan}\left(\frac{y_{e} \cdot \cos \left(\Phi_{e}\right)+z_{e} \cdot \sin \left(\Phi_{e}\right)}{x_{e}}\right)
\end{aligned}
$$

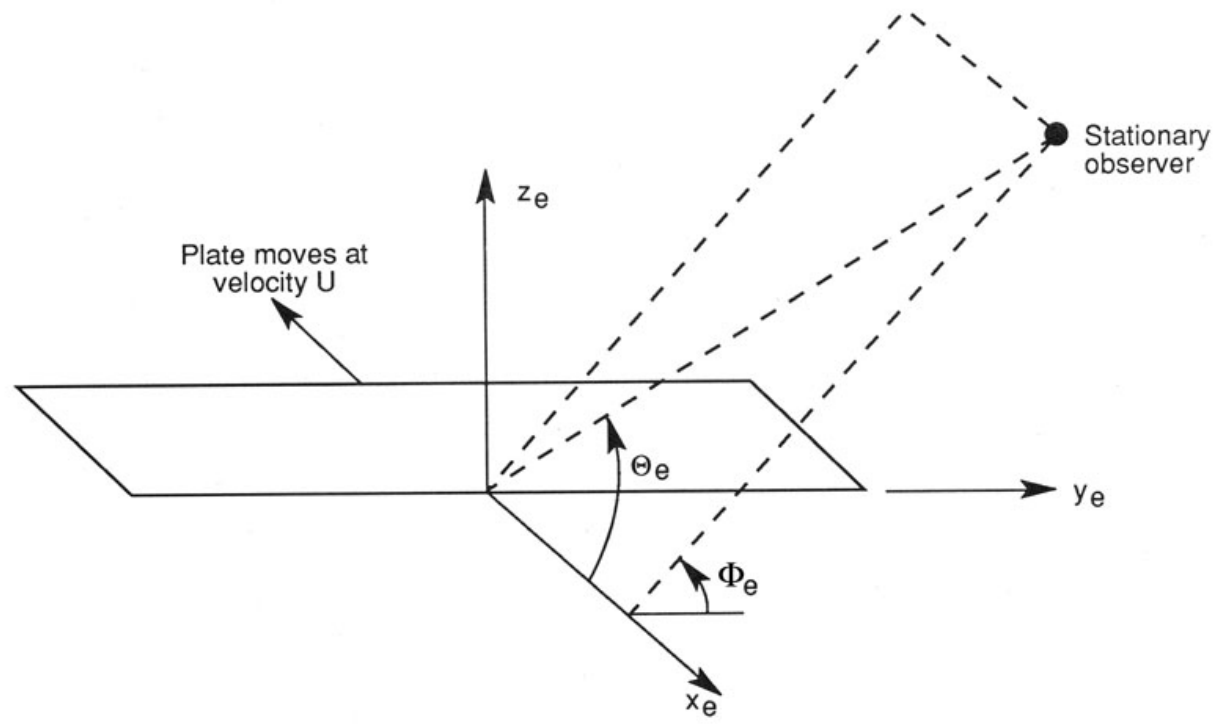

Figure 1. Angles used in the directivity function (Brooks et al. 1989; Moriarty and Migliore 2003)

The reference axis is located at each blade node and $\mathrm{x}_{e}$ is aligned with the chord, $\mathrm{y}_{e}$ is aligned with the span pointing to the blade tip, and $\mathrm{z}_{e}$ is aligned toward the airfoil suction side. Note that in OpenFAST the local airfoil-oriented reference system is used, and a rotation is applied.

Given the angles $\Theta_{e}$ and $\Phi_{e}$, at high frequency, $\bar{D}$ takes the expression:

$$
\bar{D}_{h}\left(\Theta_{e}, \Phi_{e}\right)=\frac{2 \sin ^{2}\left(\frac{\Theta_{e}}{2}\right) \sin ^{2} \Phi_{e}}{\left(1+M \cos \Theta_{e}\right)\left(1+\left(M-M_{c}\right) \cos \Theta_{e}\right)^{2}}
$$

where $M_{c}$ represents the Mach number past the trailing edge and that is here for simplicity assumed equal to $80 \%$ of free-stream $\mathrm{M}$. At low frequency, the equation becomes: 


$$
\bar{D}_{l}\left(\Theta_{e}, \Phi_{e}\right)=\frac{\sin ^{2} \Theta_{e} \sin ^{2} \Phi_{e}}{\left(1+M \cos \Theta_{e}\right)^{4}}
$$

Each model distinguishes a different value between low and high frequency. For the TI noise model, the shift between low and high frequency is defined based on $\bar{k}_{1}$. For the TBL-TE noise, the model differences instead shift between below and above stall, where $\bar{D}_{h}$ and $\bar{D}_{l}$ are used, respectively.

\subsection{A-Weighting}

The code offers the possibility to weigh the aeroacoustics outputs by A-weighting, which is an experimental coefficient that aims to take into account the sensitivity of human hearing to different frequencies. The A-weight, $A_{w}$, is computed as:

$$
\begin{aligned}
A_{w}=\frac{10 \log \left(1.562339 \frac{f^{4}}{\left(f^{2}+107.65265^{2}\right)\left(f^{2}+737.86223^{2}\right)}\right)}{\log 10} & \frac{10 \log \left(2.422881 e 16 \frac{f^{4}}{\left(f^{2}+20.598997^{2}\right)^{2}\left(f^{2}+12194.22^{2}\right)^{2}}\right)}{\log 10}
\end{aligned}
$$

The A-weighting is a function of frequency and is added to the values of sound pressure levels:

$$
S P L_{A_{w}}=S P L+A_{w}
$$




\section{Model Verification}

\subsection{Reference Wind Turbine}

The noise model of OpenFAST is exercised by simulating the aeroacoustics noise emissions of the IEA Wind Task 37 land-based reference wind turbine (Bortolotti et al. 2019). The main characteristics of the reference wind turbine are presented in Table 1.

Table 1. Main Characteristics of the IEA Wind Task 37 Land-Based Reference Wind Turbine

\begin{tabular}{|c|c|c|c|}
\hline Data & Value & Data & Value \\
\hline Wind class & $\begin{array}{c}\text { International } \\
\text { Electrotechnical } \\
\text { Commission } 3 \mathrm{~A}\end{array}$ & $\begin{array}{l}\text { Rated electrical } \\
\text { power }\end{array}$ & 3.37 megawatts \\
\hline $\begin{array}{l}\text { Rated aerodynamic } \\
\text { power }\end{array}$ & 3.6 megawatts & $\begin{array}{c}\text { Drivetrain \& } \\
\text { generator efficiency }\end{array}$ & $93.60 \%$ \\
\hline Rotor diameter & 130 meters & Hub height & 110 meters \\
\hline Cut-in wind speed & 4 meters/second & Cut-out wind speed & 25 meters/second \\
\hline Rotor cone angle & 3 degrees & Nacelle tilt angle & 5 degrees \\
\hline Max blade tip speed & 80 meters/second & $\begin{array}{l}\text { Rated tip-speed } \\
\text { ratio }\end{array}$ & 8.16 \\
\hline $\begin{array}{l}\text { Maximum } \\
\text { aerodynamic } \mathrm{Cp}\end{array}$ & 0.481 & Rated rotor speed & $\begin{array}{l}11.75 \text { revolutions per } \\
\text { minute }\end{array}$ \\
\hline
\end{tabular}

The OpenFAST model of the wind turbine is available at https://github.com/OpenFAST/r-test and is optionally coupled to the Reference OpenSource Controller. ${ }^{2}$

\subsection{Code-to-Code Comparison}

A detailed code-to-code comparison was conducted to verify the implementation of the noise models linked to OpenFAST with the implementation available at the Wind Energy Institute of the Technical University of Munich, Germany. The latter is described in Sucameli et al. (2018) and is implemented in the wind turbine design framework Cp-Max, which adopts the multibodybased aeroservoelastic solver Cp-Lambda.

The comparison is conducted for the main noise sources - turbulent inflow and the TBL-TE noise - for both the single airfoil profile and full turbine. This helped resolve a few implementation mistakes and small inconsistencies. The comparison is performed with a steady wind of 8 meters per second $(\mathrm{m} / \mathrm{s})$, no shear, a rated pitch angle of 1.17 degrees $(\mathrm{deg})$, and a fixed rotor speed of 10.04 revolutions per minute (rpm). A fixed value of 0.1 is assumed for the incident turbulent intensity, $I_{1}$.

${ }^{2}$ https://github.com/NREL/ROSCO 
Figure 2 shows the predictions in terms of SPL for the Amiet model with the angle-of-attack correction from OpenFAST, the Simplified Guidati model generated by OpenFAST, and the Amiet model from Cp-Max.

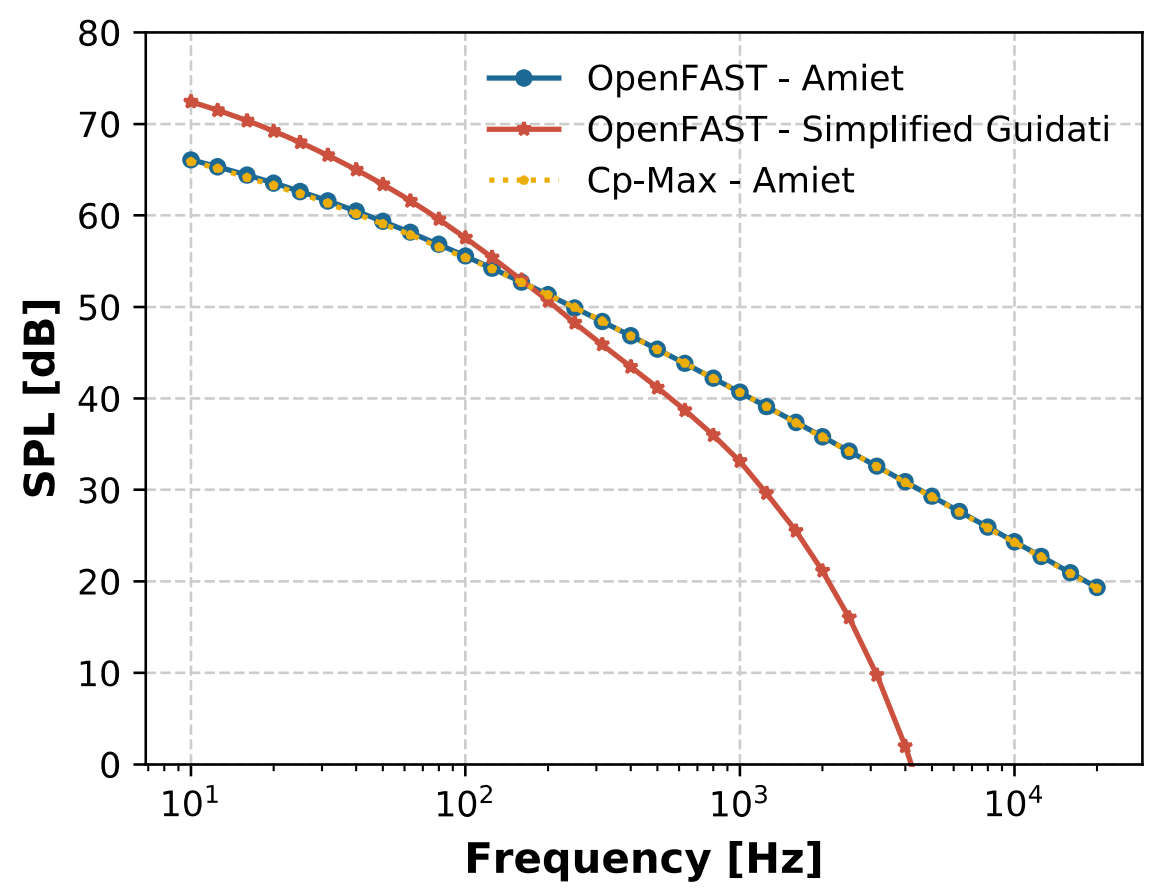

Figure 2. Code-to-code comparison for the turbulent inflow models

The two implementations of the turbulent inflow Amiet model return a perfect match between OpenFAST and Cp-Max. The chosen scenario sees the blade operating at optimal angles of attack and, therefore, the effect of the angle of attack correction is negligible. The plots also show the great difference between the Amiet model and the Simplified Guidati model. It may be useful to keep in mind that the Simplified Guidati model has, in the past, been corrected with a factor of $+10 \mathrm{~dB}$, which is applied here.

For the same inflow and rotor conditions, the BPM and TNO TBL-TE noise models are compared in Figure 3. The match is again satisfactory, although slightly larger differences emerge that are attributed to differences in the angles of attack between the two aeroelastic solvers and in different integration schemes in the TNO formulations. 


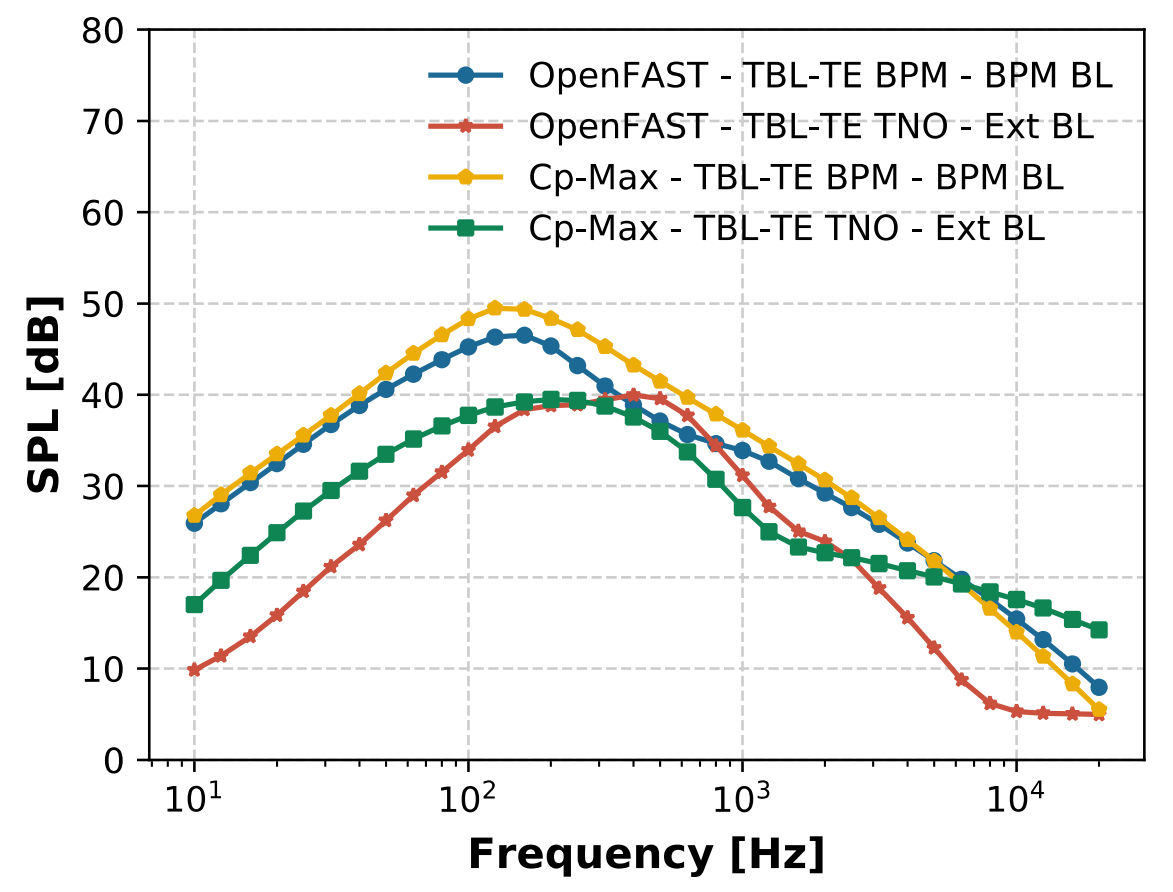

Figure 3. Code-to-code comparison for the BPM and TNO TBL-TE models. The boundary layer properties are estimated from either the BPM model (BPM BL) or defined by the user (Ext BL)

The last comparison looked at the directivity models and the overall sound pressure levels at various observer locations. Simulations are run distributing 200 observers in a horizontal square of 500 meters $(\mathrm{m})$ by $500 \mathrm{~m}$ (see Figure 4). The noise is computed from the Amiet and the BPM turbulent boundary-layer-trailing-edge models. The code-to-code comparison returns similar predictions between OpenFAST and Cp-Max. The comparison is shown in Figure 5.

The main conclusion of this code-to-code comparison is that, to the best of authors' knowledge, the models are now implemented correctly and generate similar SPL and overall SPL levels for any arbitrary observer. Nonetheless, it is clear that all of the presented models are imperfect, and improvements could be made both at the theoretical and at the implementation levels. 


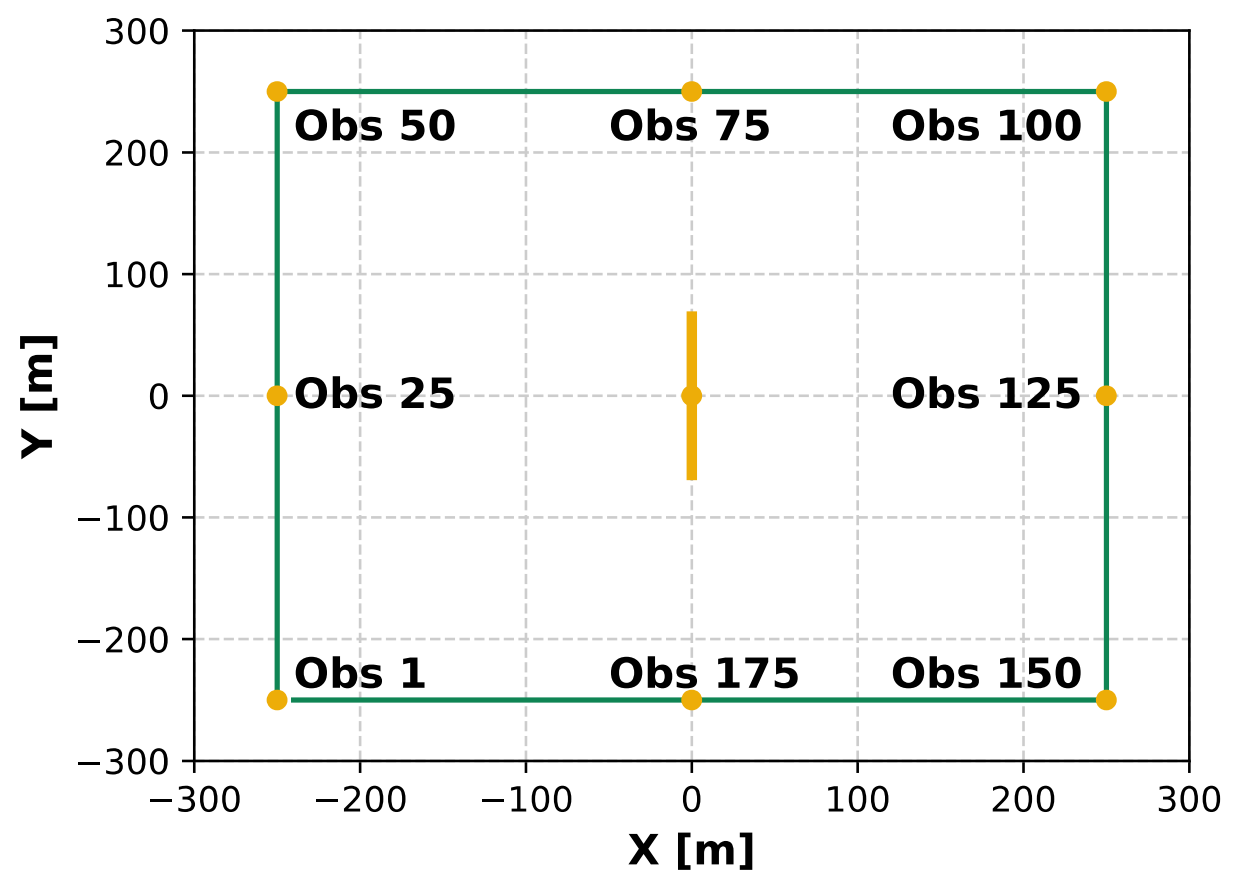

Figure 4. Location and numbering of the observers

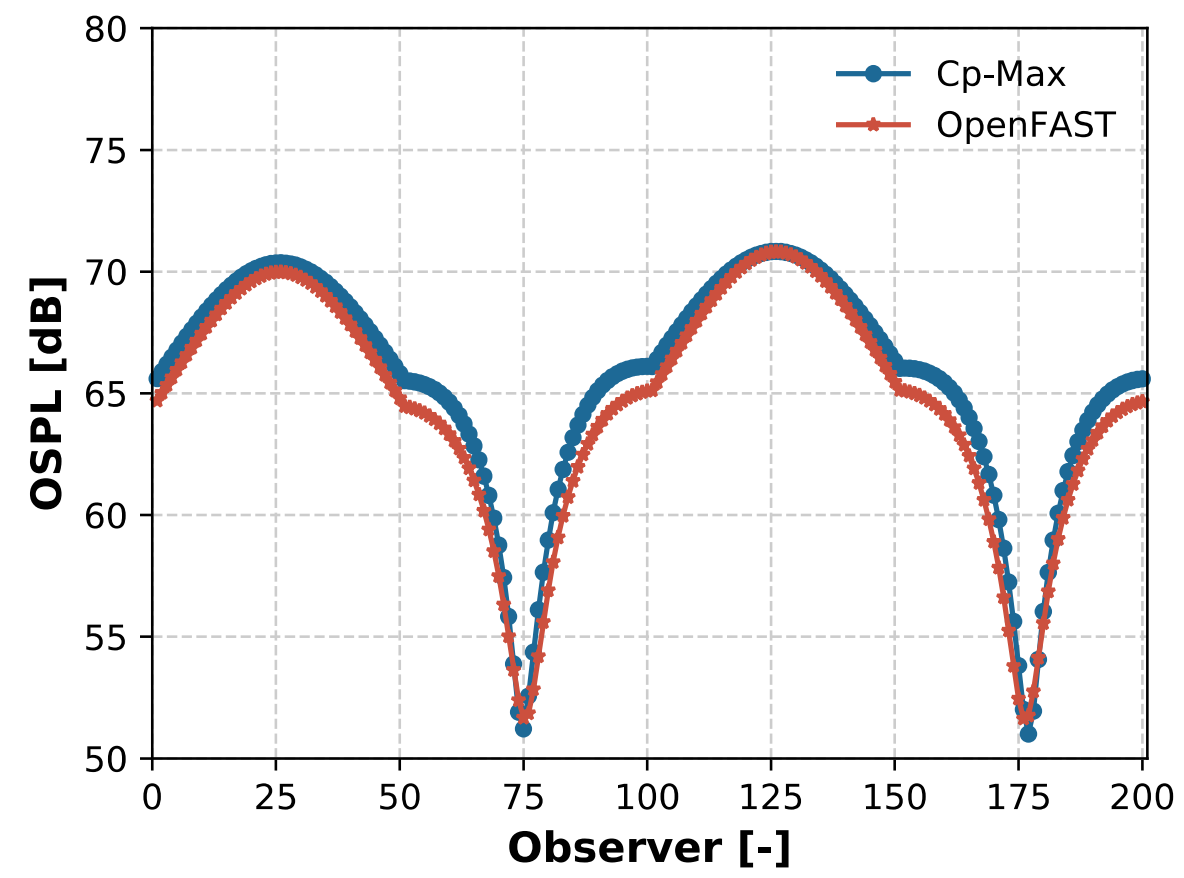

Figure 5. Comparison of overall sound pressure levels for the observers shown in Figure 4 


\subsection{Model Usage}

The aeroacoustics model of OpenFAST has four options for the outputs:

1. Overall sound pressure level (dB/A-weighted decibels [dBA]) —one value per time step per observer is generated

2. Total sound pressure level spectra $(\mathrm{dB} / \mathrm{dBA})$ — one spectrum per time step per observer is generated between $10 \mathrm{~Hz}$ and $20 \mathrm{kHz}$

3. Mechanism-dependent sound pressure level spectra $(\mathrm{dB} / \mathrm{dBA})$ - one spectrum per active noise mechanism per time step per observer is generated between $10 \mathrm{~Hz}$ and $20 \mathrm{kHz}$.

4. Overall sound pressure level (dB/A-weighted decibels $[\mathrm{dBA}])$ —one value per blade per node per time step per observer is generated.

The overall SPL from the first option can be used to plot directivity maps of the noise. An example, which was generated using a Python script, ${ }^{3}$ is shown in Figure 6. The noise map, which shows the overall SPL averaged over 1 rotor revolution, is generated for a steady wind speed of $8 \mathrm{~m} / \mathrm{s}$, a fixed rotor speed of $10.04 \mathrm{rpm}$, and a 1.17-deg pitch angle. In a horizontal circle of $500 \mathrm{~m}$ in diameter, 1681 observers are placed at a 2-m height. Only the Simplified Guidati and the BPM TBL-TE noise models are activated.

\footnotetext{
${ }^{3}$ https://github.com/OpenFAST/python-toolbox
} 


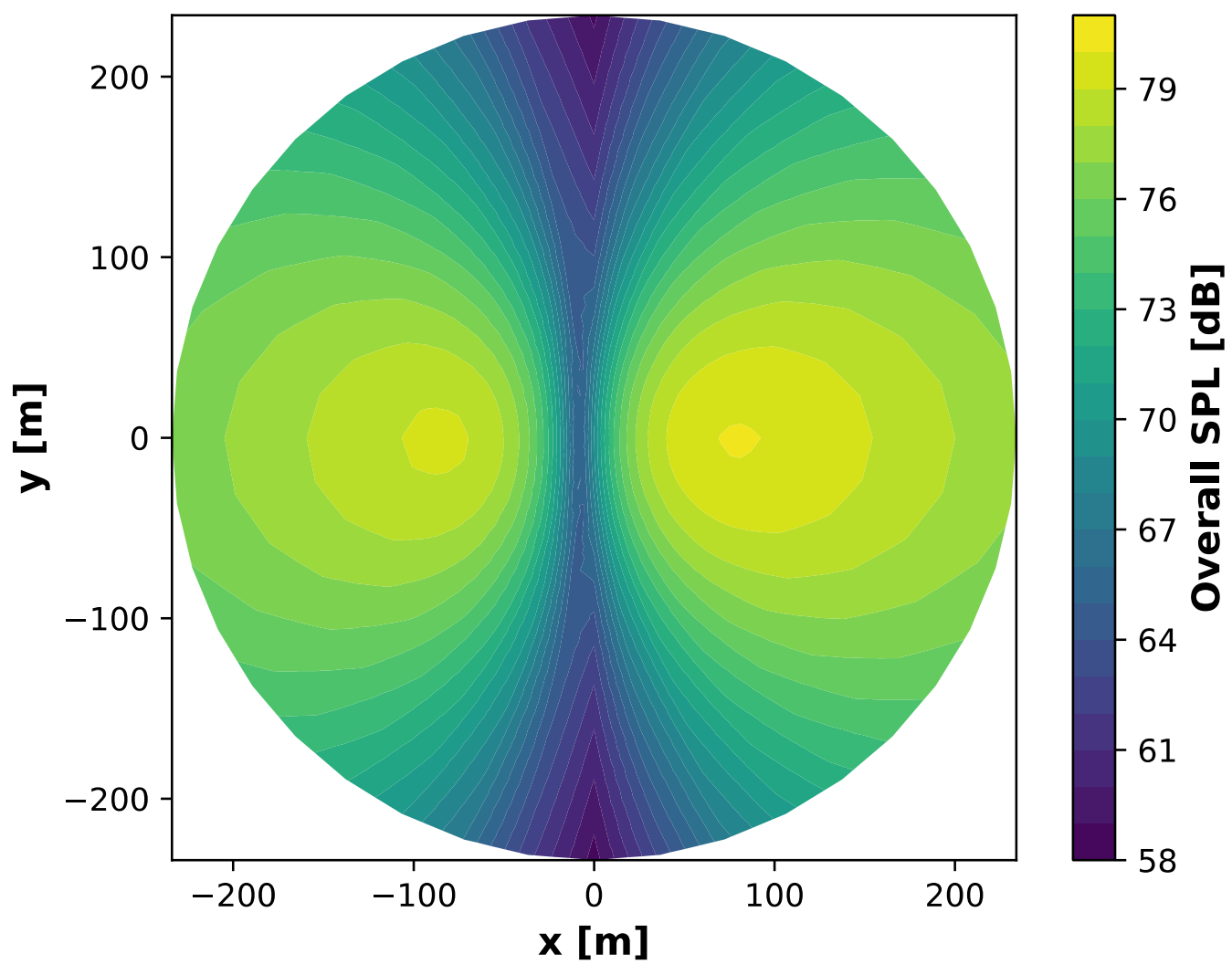

Figure 6. Map of the overall SPL of the reference wind turbine at a 2-m height from Simplified Guidati and BPM TBL-TE noise models. The wind turbine is located at $x=0, y=0$. A steady wind of 8 $\mathrm{m} / \mathrm{s}$ blows from left $(-x)$ to right $(+x)$.

The second output can be used to generate SPL spectra. These spectra can be computed for various observers and optionally A-weighted to account for human hearing. Figure 7 shows the total SPL spectra computed for the same rotor conditions of the previous example. The A-weight greatly reduces the curve at frequency below $1,000 \mathrm{~Hz}$ while slightly increasing those between $1 \mathrm{kHz}$ and $8 \mathrm{kHz}$. 


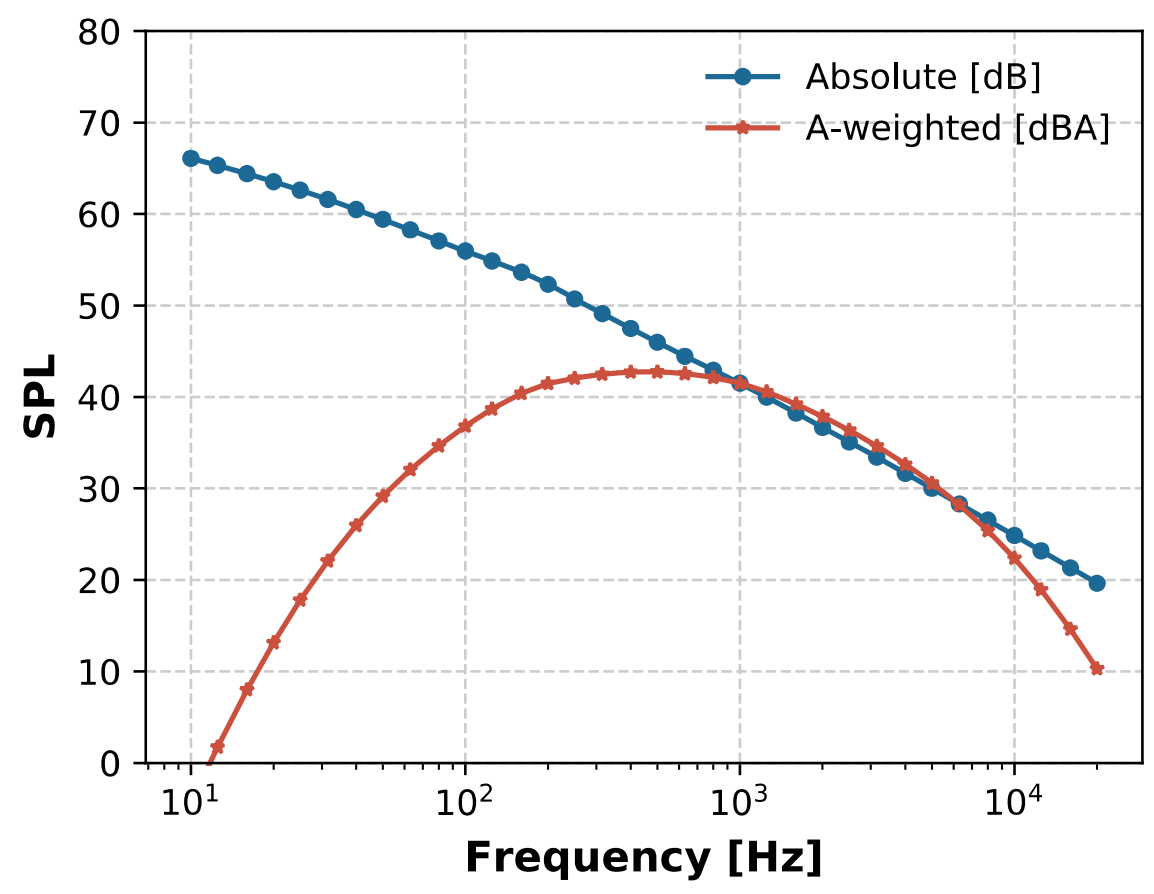

Figure 7. Comparison between absolute and A-weighted SPL

The third output distinguishes the SPL spectrum per mechanism. Figure 8 shows the various SPL spectra estimated by each noise model for the same rotor conditions reported earlier. The total spectrum is visibly dominated by the turbulent inflow, TBL-TE, and trailing-edge bluntness noise mechanisms. Notably, the latter is extremely sensitive to its inputs, $\Psi$ and $h$. The reference wind turbine is a purely numerical model, and these quantities have been arbitrarily set. Users should pay attention to these inputs when calling the trailing-edge bluntness model. Consistent with literature, the laminar boundary layer-vortex shedding and tip vortex noise mechanisms have negative $\mathrm{dB}$ values and are, therefore, not visible.

Notably, these spectra are not A-weighted, but users can activate the flag and obtain A-weighted spectra. 


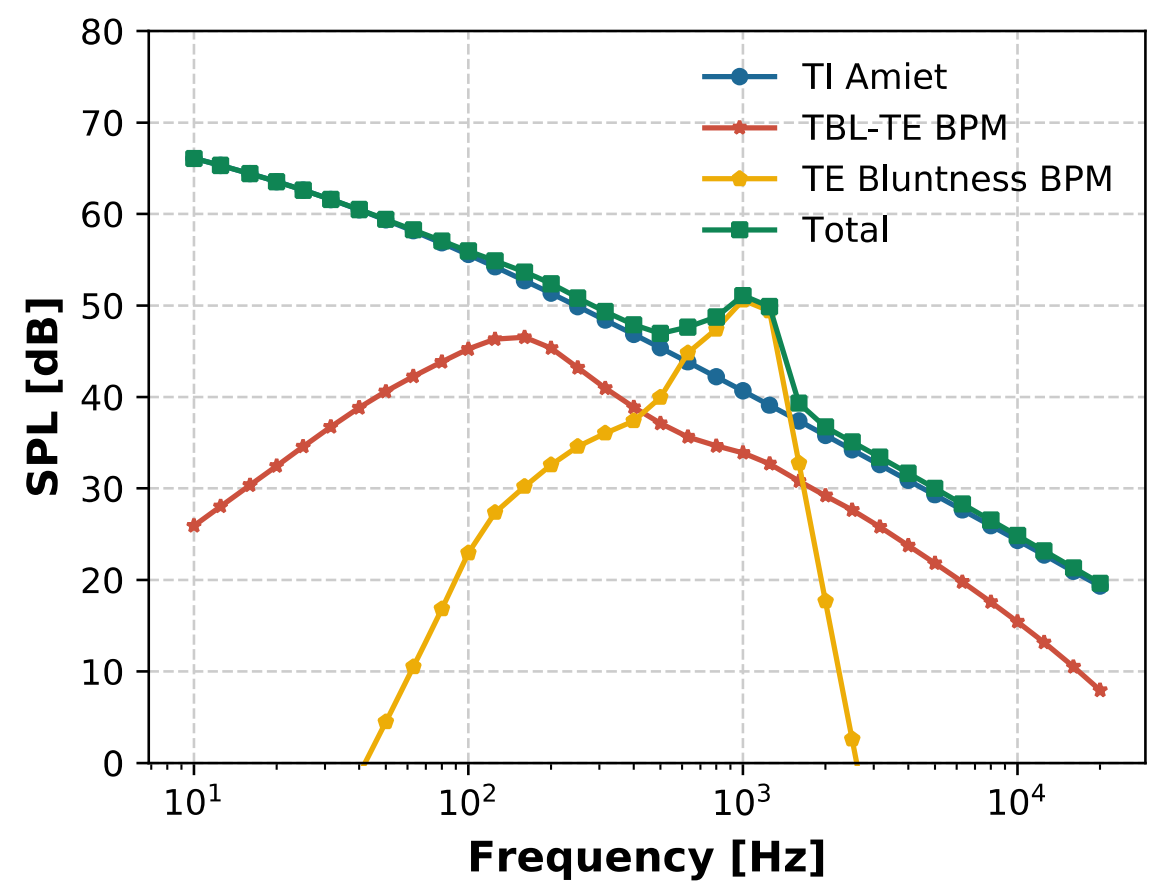

Figure 8. Nonweighted SPL spectra of the various noise mechanisms

Finally, the fourth output can be used to visualize the noise emission across the rotor. Figure 9 shows the noise generation of the rotor as seen from an observer located $175 \mathrm{~m}$ downwind at a height of $2 \mathrm{~m}$. The map is generated by plotting the overall SPL generated by one blade during one rotor revolution. The plot shows that higher noise is observed when the blade is descending (the rotor from behind is seen rotating counterclockwise). This effect, which matches the results shown in Moriarty and Migliore (2003), is explained by the asymmetry of Eq. 31. Noise is indeed higher when the observer faces the leading edge of an airfoil (high $\Theta_{e}$ ) than the trailing edge $\left(\right.$ low $\left.\Theta_{e}\right)$. 


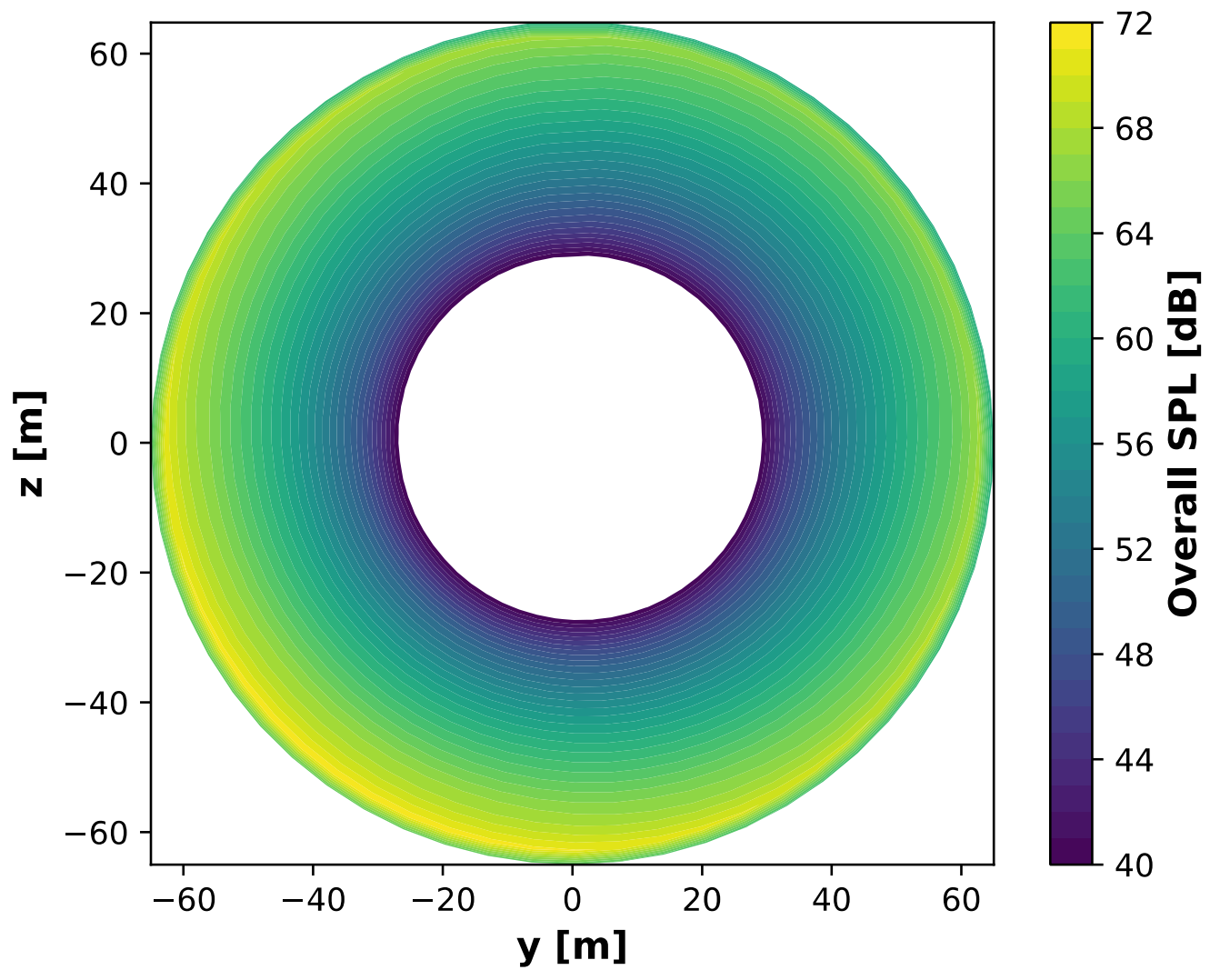

Figure 9. Map of the overall SPL of the rotor of the reference wind turbine from the Simplified Guidati and BPM TBL-TE noise models. The observer is located $175 \mathrm{~m}$ downwind at a height of 2 m. 


\section{Conclusions}

This document describes a set of frequency-based aeroacoustics models coupled to the opensource aeroservoelastic solver OpenFAST. The goal of these models is to predict the aeroacoustics emissions of wind turbine rotors. The document shows a code-to-code comparison between the models coupled to OpenFAST and the models implemented at the Technical University of Munich and coupled to the aeroservoelastic solver Cp-Lambda. The comparison is performed simulating the aeroacoustics emissions of the IEA Wind Task 37 land-based reference wind turbine. The results show a good agreement between the two implementations. The same turbine model is later used to exercise the aeroacoustics model showcasing its capabilities. Finally, the appendices describe the entries of the input files of OpenFAST to run the aeroacoustics analysis.

Future work will focus on validating the aeroacoustics models. In parallel, propagation models will be investigated and implemented. Finally, attention will be dedicated to infrasound noise and to the time-domain models that can simulate it. 


\section{References}

Amiet, Roy K. 1975. "Acoustic Radiation from an Airfoil in a Turbulent Stream." Journal of Sound and Vibration 41(4): 407-420. https://doi.org/10.1016/S0022-460X(75)80105-2.

Bortolotti, Pietro, Helena Canet Tarres, Katherine Dykes, Karl Merz, Latha Sethuraman, David Verelst, and Frederik Zahle. 2019. Systems Engineering in Wind Energy - WP2.1 Reference Wind Turbines. IEA Technical Report. https://www.nrel.gov/docs/fy19osti/73492.pdf.

Brooks, Thomas F., D. Stuart Pope, and Michael A. Marcolini. 1989. "Airfoil Self-Noise and Prediction." NASA Reference Publication 1218.

Drela, Mark, and Michael B. Giles. 1987. "Viscous-inviscid analysis of transonic and low Reynolds number airfoils." AIAA Journal 25(10). https://doi.org/10.2514/3.9789.

Guidati, Gianfranco, Rainer Bareiss, Siegfried Wagner, Ton Dassen, and Rene Parchen. 1997. "Simulation and Measurement of Inflow-Turbulence Noise on Airfoils." $3^{\text {rd }}$ AIAA/CEAS Aeroacoustics Conference. https://doi.org/10.2514/6.1997-1698.

Lowson, Martin V. 1970. "Theoretical Analysis of Compressor Noise Evaluation.” The Journal of the Acoustical Society of America 47: 371-385. https://doi.org/10.1121/1.1911508.

Klein, Levin, Jonas Gude, Florian Wenz, Thorsten Lutz, and Ewald Krämer. 2018. "Advanced Computational Fluid Dynamics (CFD)-Multi-Body Simulation (MBS) Coupling to Assess LowFrequency Emissions from Wind Turbines." Wind Energy Science Journal 3: 713-728. https://doi.org/10.5194/wes-3-713-2018.

Moriarty, Patrick J., and Paul G. Migliore. 2003. Semi-Empirical Aeroacoustic Noise Prediction Code for Wind Turbines (Technical Report). Golden, CO: National Renewable Energy Laboratory. NREL/TP-500-34478. https://www.nrel.gov/docs/fy04osti/34478.pdf.

Moriarty, Patrick J., Gianfranco Guidati, and Paul Migliore. 2004. "Recent Improvement of a Semi-Empirical Aeroacoustic Prediction Code for Wind Turbines." $10^{\text {th }}$ AIAA/CEAS Aeroacoustics Conference. https://doi.org/10.2514/6.2004-3041.

Moriarty, Patrick J., Gianfranco Guidati, and Paul Migliore. 2005. "Prediction of Turbulent Inflow and Trailing-Edge Noise for Wind Turbines." $11^{\text {th }}$ AIAA/CEAS Aeroacoustics Conference. https://doi.org/10.2514/6.2005-2881.

Moriarty, Patrick J., and A. C. Hansen. 2005. AeroDyn Theory Manual (Technical Report). Golden, CO: National Renewable Energy Laboratory. NREL/TP-500-36881. https://www.nrel.gov/docs/fy05osti/36881.pdf.

Moriarty, Patrick J. 2005. "NAFNoise User's Guide.” https://github.com/NREL/NAFNoise/blob/master/NAFNoise.pdf.

OpenFAST GitHub Repository. https://github.com/OpenFAST/openfast. dev branch, July 2020. 
Parchen, René R. 1998. "Progress Report DRAW: A Prediction Scheme for Trailing Edge Noise Based on Detailed Boundary Layer Characteristics." TNO Institute of Applied Physics.

Paterson, Robert W., and Roy K. Amiet. 1976. "Acoustic radiation and surface pressure characteristics of an airfoil due to incident turbulence." $3^{\text {rd }}$ AIAA Aero-Acoustics Conference, Palo Alto, CA.

Sucameli, Carlo R., Pietro Bortolotti, Alessandro Croce, Carlo L. Bottasso. 2018. "Comparison of Some Wind Turbine Noise Emission Models Coupled to BEM Aerodynamics." Journal of Physics: Conference Series 1037. https://doi.org/10.1088/1742-6596/1037/2/022038.

Viterna, Larry A. 1981. "Method for Predicting Impulsive Noise Generated by Wind Turbine Rotors." DOE/NASA/20320-36.

https://ntrs.nasa.gov/archive/nasa/casi.ntrs.nasa.gov/19820013840.pdf.

Zhu, Wei J., Nicolai Heilskov, Wen Zhong Shen. 2005. "Modeling of Aerodynamically Generated Noise From Wind Turbines." Journal of Solar Energy Engineering 127(4): 517-528. https://doi.org/10.1115/1.2035700 


\section{Appendix A. Call the Aeroacoustics Model}

A live version of this documentation is available at https://openfast.readthedocs.io/. To run the aeroacoustics model, the flag CompAA needs to be set to True in the AeroDyn15 main input file in the inputs block General Options. When the flag is set to True, the following line must include the name of the file containing the inputs to the aeroacoustics model, which is discussed in Appendix A.2.

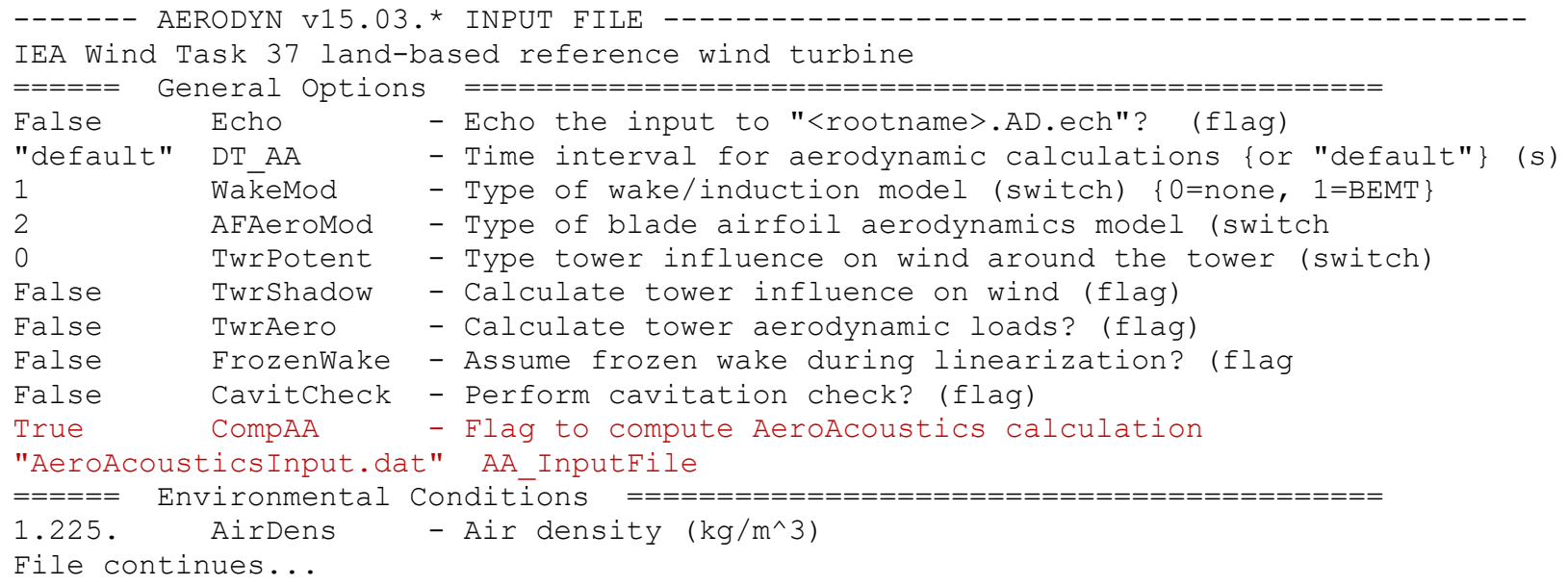




\section{A.1 Main Input File}

The aeroacoustics main input file comprises a series of inputs and flags that should be set appropriately depending on the analysis that should be run. These are split into the subfields General Options, Aeroacoustics Models, Observer Input, and Outputs.

Starting from the General Options, these are:

- Echo-True/False: option to rewrite the input file with the correct template

- $D T \_A A-F l o a t:$ time step of the aeroacoustics computations. Only multiples of the time step DTAero of AeroDyn can be used. If set to default, the time step DTAero is adopted.

- AAStart - Float: time after which the aeroacoustics module is run.

- BldPrent - Float: percentage value of blade span measured from blade tip that contributes to the noise emissions; $100 \%$ corresponds to the entire blade.

The field Aeroacoustics Models lists all the flags for the actual noise models:

- TIMod - Integer 0/1/2: flag to set the turbulent inflow noise model; 0 turns it off, 1 corresponds to the Amiet model discussed in Section 2.1.1, and 2 corresponds to the Simplified Guidati model presented in Section 2.1.2.

- TICalcMeth - Integer 1/2: flag to set the calculation method for the incident turbulence intensity. When set to 1 , incident turbulence intensity is defined in a userdefined grid; see Appendix A.4. When set to 2, incident turbulence intensity is estimated from the time history of the incident flow.

- TICalcTabFile-String: name of the text file with the user-defined turbulence intensity grid; see Appendix A.4.

- SurfRoughness - Float: value of $z_{0}$ used to estimate $L_{t}$ in the Amiet model.

- TBLTEMod - Integer 0/1/2: flag to set the TBL-TE noise model; 0 turns off the model, 1 uses the Brooks-Pope-Marcolini (BPM) airfoil noise model (see Section 2.2.1), and 2 uses the TNO model described in Section 2.2.2.

- BLMod - Integer 1/2: flag to set the calculation method for the boundary layer characteristics; 1 uses the simplified equations from the BPM model, 2 loads the files as described in Appendix A.2. Only used if TBLTEMod is different than zero.

- TripMod - Integer 0/1/2: if BLMod is set to 1, different semiempirical parameters are used for a nontripped boundary layer (TRipMod $=0$ ), heavily tripped boundary layer (TRipMod=1), or lightly tripped boundary layer (TRipMod=2); 2 is typically used for operational wind turbines, whereas 1 is often used for wind tunnel airfoil models. 
- LamMod - Integer 0/1: flag to activate the laminar boundary layer - vortex shedding model, presented in Section 2.3.

- TipMod - Integer 0/1: flag to activate the tip vortex model, presented in Section 2.4.

- RoundedTip - True/False: if TipMod=1, this flag switches between a round tip (True) and a square tip (False), see Section 2.4.

- Alprat - Float: value of the slope of the lift coefficient curve at blade tip; see Section 2.4 .

- BluntMod - Integer 0/1: flag to activate (BluntMod=1) the trailing-edge bluntness vortex shedding model, see Section 2.5. If the flag is set to 1, the trailing-edge geometry must be specified in the file(s) listed in the field Blade Properties.

Next, the field Blade Properties lists three file names, often but not necessarily identical, which contain the distributed properties describing the detailed geometry of the trailing edge. These are described in Appendix A.5.

The field ObserverLocations contains the path to the file where the number of observers (NrObsLoc) and the respective locations are specified; see Appendix A.3.

Finally, the set Outputs contains a few options for the output data:

- AWeighting - True/False: flag to set whether the sound pressure levels are reported with (True) or without (False) the A-weighting correction; see Section 3.3.

- NAAOutFile - Integer 1/2/3/4: flag to set the desired output file. When set to 1 , a value of overall sound pressure level at every DT_AA time step per observer is printed to file. When set to 2, the first output is accompanied by a second file where the total sound pressure level spectrum is printed per time step per observer. When set to 3, the two first outputs are accompanied by a third file where the sound pressure level spectrum per noise mechanism is printed per time step per observer. When set to 4 , a fourth file is generated with the values of overall sound pressure levels per node, per blade, per observer, and per time step.

- The following line contains the file name used to store the outputs. The file name is attached with a 1, 2, 3, and 4 flag based on the NAAOutFile options.

The file must be closed by an END command. 
AeroAcoustics Model v1.00.* INPUT FILE

Row for Turbine Name and characteristics.

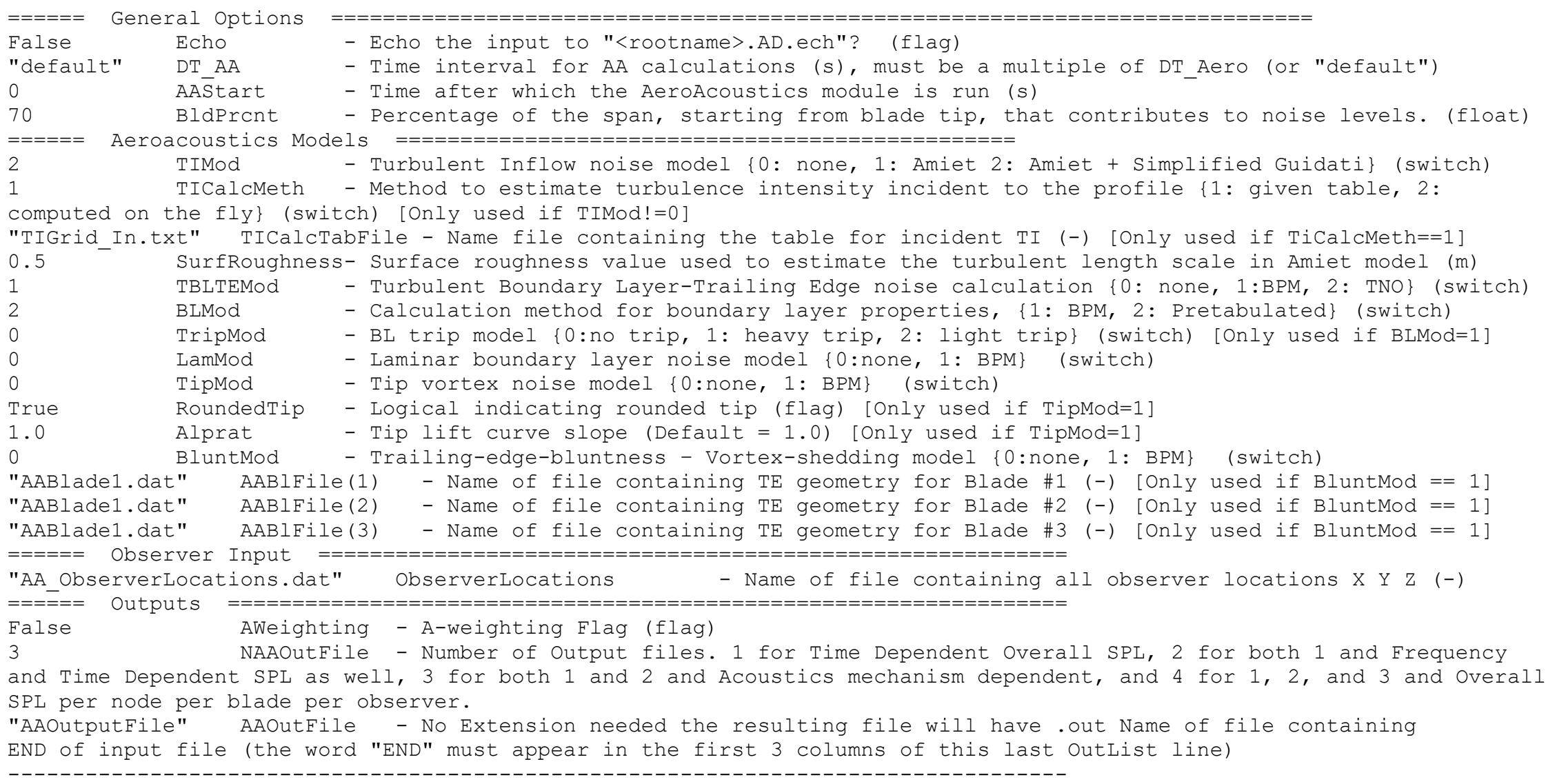




\section{A.2 Boundary Layer Inputs}

When the flag BLMod is set equal to 2, pretabulated properties of the boundary layer must be provided and are used by the turbulent boundary layer - trailing-edge noise models. The file name is to be specified in the field BL_file among the inputs of the file with the airfoil polar coefficients. One airfoil file must be specified per aerodynamic station.

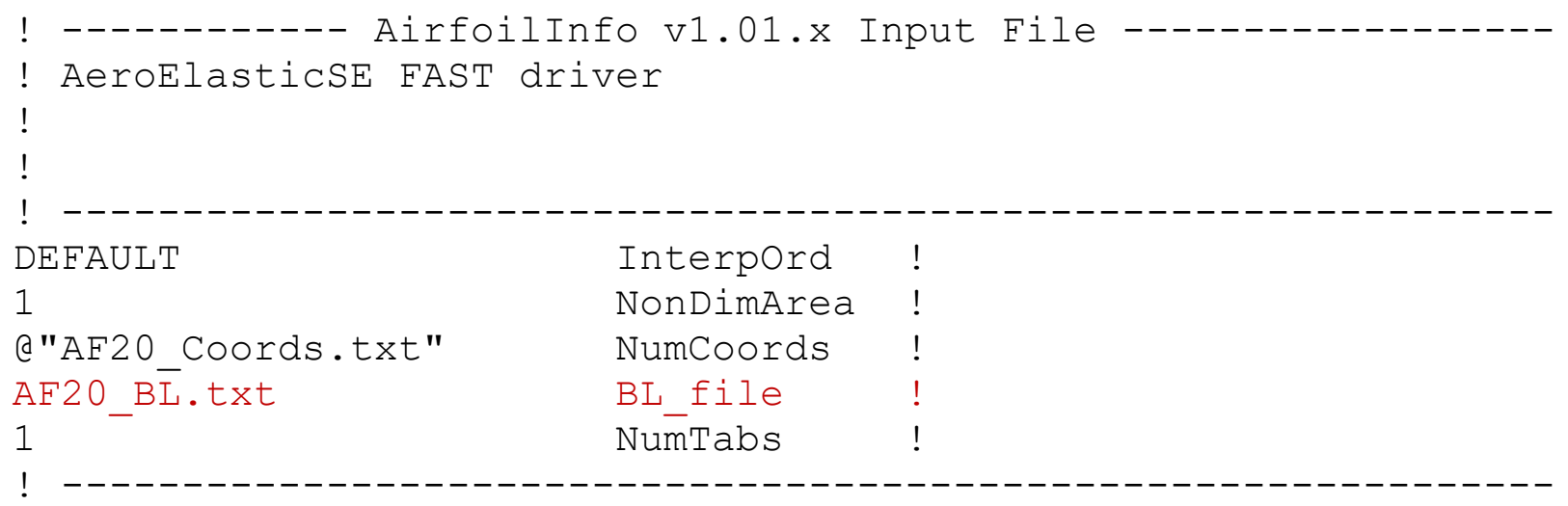

The file, in this example named AF20_BL.txt, contains 8 inputs, which are tabulated for a given number of Reynolds numbers, ReListBL, and a given number of angles of attack, aoaListBL. The inputs, which are defined nondimensionally and must be provided for the suction and pressure side of the airfoil above and below the trailing edge, are:

- Ue_Vinf - flow velocity at the top of the boundary layer

- Dstar $-\delta^{*}$, boundary layer displacement thickness

- Delta $-\delta$, nominal boundary layer thickness

- $\mathrm{Cf}$ - friction coefficient.

In the following example, the file was generated thanks to a Python script ${ }^{4}$ that runs the boundary layer solver, XFoil. Notably, XFoil, by default, does not return $\delta$, but the boundary layer momentum thickness, $\theta . \delta$ can be reconstructed using the expression from Drela and Giles (1987):

$$
\delta=\theta \cdot\left(3.15+\frac{1.72}{H-1}\right)+\delta^{*}
$$

where $H$ is the kinematic shape factor, which is also among the standard outputs of XFoil. Because it is usually impossible to obtain these values for the whole ranges of Reynolds numbers and angles of attack, the code is set to adopt the nearest available values and print to screen a warning.

\footnotetext{
${ }^{4}$ https:/github.com/OpenFAST/python-toolbox
} 
! Boundary layer characteristics at the trailing edge for the airfoil coordinates of AF20_Coords.txt

! Legend: aoa - angle of attack (deg), Re - Reynolds number (-, millions), PS - pressure side, SS - suction side, Ue Vinf - edge velocity (-), Dstar - displacement thickness (-), Delta - nominal boundary layer thickness (-) Cf - friction coefficient (-)

4 RelistBL - Number of Reynolds numbers (it corresponds to the number of tables)

30 aoalistBL - Number of angles of attack (it corresponds to the number of rows in each table) $0.50-\operatorname{Re}$

aoa

(deg)

$-5.00000$

$-3.96552$

$-1.89655$

$-0.86207$

0.17241

1.20690

2.24138

3.27586

4. 31034

5.34483

6.37931

7.41379

8.44828

9.48276

10.51724

11.55172

12.58621

13.62069

14.65517

15.68966

16.72414

17.75862

18.79310

19.82759

20.86207

21.89655

22.93103

23.96552

25.00000

1.00

aoa

(deg)

$-5.00000$

$-3.96552$

$-2.93103$

$-1.89655$

$-0.86207$

0.17241

1.20690

2.24138

3.27586

4.31034
Ue_Vinf_SS $(-)$

$8.39390 e-01$

$8.42050 e-01$

$8.45320 e-01$

$8.48230 e-01$

$8.51550 e-01$

$8.55000 \mathrm{e}-01$

$8.63820 e-01$

$8.61500 e-01$

$8.64430 e-01$

$8.67960 e-01$

$8.72300 e-01$

$8.77930 \mathrm{e}-01$

$8.86840 e-01$

$9.00620 e-01$

$9.20300 e-01$

$9.48080 e-01$

$9.89560 e-01$

$1.02883 e+00$

$1.05789 e+00$

$1.07975 e+00$

$1.09657 \mathrm{e}+00$

$1.11040 e+00$

$1.12290 \mathrm{e}+00$

$1.13461 e+00$

$1.14605 e+00$

1. $16808 \mathrm{e}+00$

$1.17845 \mathrm{e}+00$

$1.18930 e+00$

$1.19987 e+00$

Re

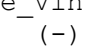

$8.34300 e-01$

$8.37330 e-01$

$8.40670 \mathrm{e}-01$

8. $44170 e-01$

$8.47840 e-01$

$8.51730 e-01$

$8.55470 e-01$

$8.59040 e-01$

$8.63480 e-01$

8. $67590 \mathrm{e}-01$

$$
\text { Ue_Vinf_PS }
$$

$$
(-)
$$

$-8.37360 e-01$

$-8.40230 e-01$

$-8.43690 e-01$

$-8.46710 e-01$

$-8.50140 \mathrm{e}-01$

$-8.53670 e-01$

$-1.04207 e+00$

$8.60210 \mathrm{e}-01$

$-8.63080 e-01$

$-8.66600 e-01$

$-8.70850 e-01$

$-8.76410 e-01$

$-8.85140 e-01$

$-8.98660 e-01$

$-9.17700 \mathrm{e}-01$

$9.44440 \mathrm{e}-01$

$-9.84930 e-01$

$-1.02353 e+00$

$-1.05226 e+00$

$-1.07394 \mathrm{e}+00$

$-1.09067 e+00$

$-1.10441 e+00$

$-1.11682 e+00$

$-1.12844 \mathrm{e}+00$

$-1.13974 e+00$

$-1.15073 e+00$

$-1.16138 e+00$

$-1.17148 e+00$

$-1.18205 e+00$

$-1.19227 e+00$

Ue_Vinf_PS

$(-)$

$-8.32480 e-01$

$-8.35790 e-01$

$-8.39370 e-01$

$-8.43070 e-01$

$-8.46890 e-01$

$-8.50900 e-01$

$-8.54730 e-01$

\begin{tabular}{|c|c|}
\hline$\underset{(-)}{\operatorname{Dstar} S S}$ & $\underset{(-\overline{)}}{\operatorname{Dstar} P S}$ \\
\hline $7.43700 e-03$ & $1.07730 \mathrm{e}-02$ \\
\hline $8.26600 e-03$ & $9.29500 e-03$ \\
\hline $9.08800 e-03$ & $8.10000 e-03$ \\
\hline $9.97400 e-03$ & $7.33700 e-03$ \\
\hline $1.09130 e-02$ & $6.54100 e-03$ \\
\hline $1.18900 \mathrm{e}-02$ & $5.92900 e-03$ \\
\hline $1.22130 e-02$ & $9.89500 e-03$ \\
\hline $1.40420 e-02$ & $4.88700 e-03$ \\
\hline $1.52900 \mathrm{e}-02$ & $4.57300 e-03$ \\
\hline $1.65660 \mathrm{e}-02$ & $4.09100 e-03$ \\
\hline $1.81000 e-02$ & $3.81700 e-03$ \\
\hline $1.98500 \mathrm{e}-02$ & $3.39700 e-03$ \\
\hline $2.22250 e-02$ & $3.15000 e-03$ \\
\hline $2.54290 e-02$ & $2.75900 e-03$ \\
\hline $2.99830 e-02$ & $2.48300 e-03$ \\
\hline $3.80160 e-02$ & $2.13200 e-03$ \\
\hline $5.83630 e-02$ & $1.85700 e-03$ \\
\hline $8.80990 e-02$ & $1.66700 e-03$ \\
\hline $1.18914 \mathrm{e}-01$ & $1.51000 e-03$ \\
\hline $1.48726 \mathrm{e}-01$ & $1.41900 e-03$ \\
\hline $1.76430 e-01$ & $1.34400 e-03$ \\
\hline $2.02883 e-01$ & $1.26100 e-03$ \\
\hline $2.29606 e-01$ & $1.20600 e-03$ \\
\hline $2.55478 e-01$ & $1.15500 e-03$ \\
\hline $2.80923 e-01$ & $1.08200 e-03$ \\
\hline $3.05117 e-01$ & $1.03800 e-03$ \\
\hline $3.27770 e-01$ & $9.81000 e-04$ \\
\hline $3.48909 e-01$ & $9.33000 e-04$ \\
\hline $3.70277 e-01$ & $8.93000 e-04$ \\
\hline $3.90503 e-01$ & $8.36000 e-04$ \\
\hline$\underset{(-)}{\operatorname{Dstar} S S}$ & $\operatorname{Dstar}_{(-)}$PS \\
\hline $6.49600 \mathrm{e}-03$ & $7.74600 e-03$ \\
\hline $7.10100 e-03$ & $6.55800 e-03$ \\
\hline $7.75600 e-03$ & $5.65600 e-03$ \\
\hline $8.45300 e-03$ & $4.96000 e-03$ \\
\hline $9.21600 e-03$ & $4.45100 e-03$ \\
\hline $1.00790 e-02$ & $3.95100 e-03$ \\
\hline $1.09340 e-02$ & $3.54400 e-03$ \\
\hline & \\
\hline & $2.91700 e-03$ \\
\hline & $2.69800 e-03$ \\
\hline
\end{tabular}

$-8.62770 \mathrm{e}-01$

$-8.66830 \mathrm{e}-01$$$
(-\overline{)}
$$

2.75094e-02

2. $98650 \mathrm{e}-02$

3. $19790 \mathrm{e}-02$

$3.44024 \mathrm{e}-02$

$3.68822 \mathrm{e}-02$

$3.96199 \mathrm{e}-02$

$4.18890 \mathrm{e}-02$

$4.51813 e-02$

$4.85938 \mathrm{e}-02$

$5.17768 e-02$

$5.43379 \mathrm{e}-02$

$5.69109 e-02$

$5.81316 \mathrm{e}-02$

$5.91946 e-02$

$6.07767 e-02$

$6.65531 \mathrm{e}-02$

$8.76076 \mathrm{e}-02$

1. $21588 \mathrm{e}-01$

1. $57264 \mathrm{e}-01$

1. $91423 e-01$

2. $22657 e-01$

2. $52158 \mathrm{e}-01$

$2.81695 e-01$

$3.10143 e-01$

$3.37970 e-01$

3. $64240 \mathrm{e}-01$

3. $88826 \mathrm{e}-01$

$4.11299 e-01$

$4.34300 e-01$

4.55921e-01

Delta_SS

2. $28566 e-02$

2. $45059 e-02$

2. $62162 e-02$

$2.79616 e-02$

2. $98142 \mathrm{e}-02$

$3.18738 \mathrm{e}-02$

$3.37289 e-02$

$3.55603 e-02$

$3.78947 e-02$

3. $97441 e-02$ $(-\overline{)}$

$5.15849 e-02$

$4.87153 e-02$

$4.70045 e-02$

$4.50456 e-02$

$4.30884 \mathrm{e}-02$

$4.27416 \mathrm{e}-02$

$1.68156 \mathrm{e}-02$

$3.93105 e-02$

3. $82233 e-02$

$3.63749 e-02$

3. $522780-02$

3. $31481 e-02$

$3.19040 e-02$

$2.95298 \mathrm{e}-02$

$2.75551 \mathrm{e}-02$

$48447 e-02$

$2.18890 e-02$

$2.00072 \mathrm{e}-02$

$1.78004 \mathrm{e}-02$

$1.65710 \mathrm{e}-02$

$1.56180 \mathrm{e}-02$

$1.43276 \mathrm{e}-02$

$1.35432 \mathrm{e}-02$

$.16844 \mathrm{e}-02$

$1.10866 \mathrm{e}-02$

$1.02373 e-02$

$9.52780 e-03$

$9.01762 \mathrm{e}-03$

$8.12755 e-03$

Delta_PS

$3.97467 e-02$

$3.67266 \mathrm{e}-02$

$3.42658 e-02$

3.22259

$3.07238 e-02$

$2.89503 e-02$

$2.74209 \mathrm{e}-02$

$2.64490 e-02$

$2.39342 e-02$
$(-)$

$.13200 e-03$

$1.04400 e-03$

9. $58000 e-04$

$8.90000 e-04$

$8.26000 e-04$

$7.79000 \mathrm{e}-04$

$8.18000 \mathrm{e}-04$

$6.78000 e-04$

$6.39000 e-04$

$5.96000 e-04$

$5.09000 e-0$

$4.18000 e-04$

2. $64000 e-04$

$1.64000 e-04$

$5.00000 e-06$

$-1.60000 e-05$

$-1.50000 e-05$

$-1.30000 e-05$

$-1.10000 e-05$

$-1.00000 e-05$

$-9.00000 e-06$

$-9.00000 e-06$

$-8.00000 e-06$

$-8.00000 e-06$

$-8.00000 e-06$

$-7.00000 e-06$

$-7.00000 e-06$

$-7.00000 e-06$

$-7.00000 e-06$

$-7.00000 e-06$

Cf_SS

$(-)$

$8.39000 e-04$

$7.84000 e-0$

$7.27000 \mathrm{e}-04$

$6.72000 e-04$

$6.180000-04$

5.65000

$.12000 \mathrm{e}-04$

$4.62000 \mathrm{e}-0$

$3.50000 e-0$

Cf_PS

$.58200 e-03$

$2.16500 e-03$

$2.35800 e-03$

$2.59900 e-03$

$2.87100 e-03$

.

$3.28700 \mathrm{e}-03$ 


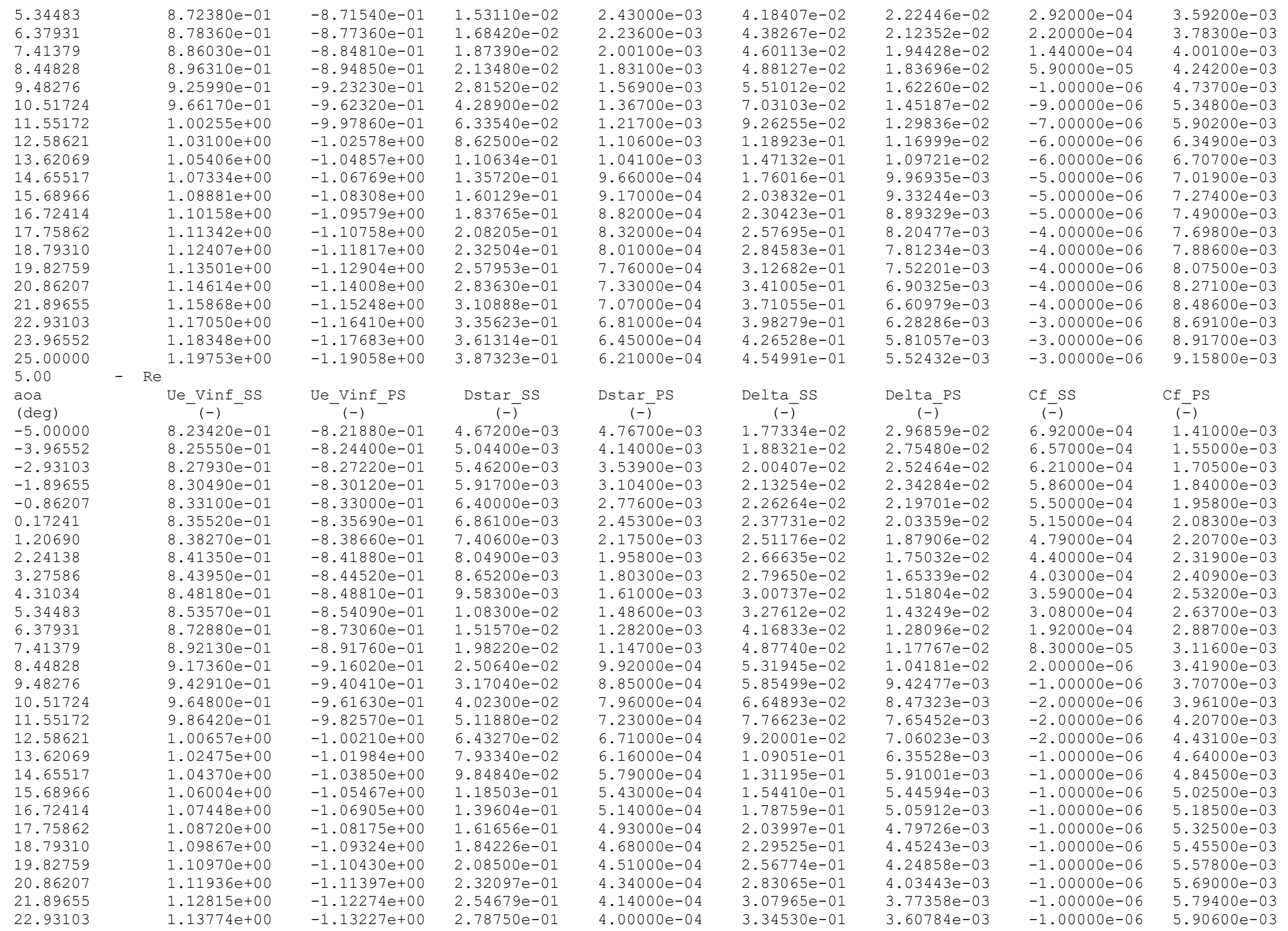




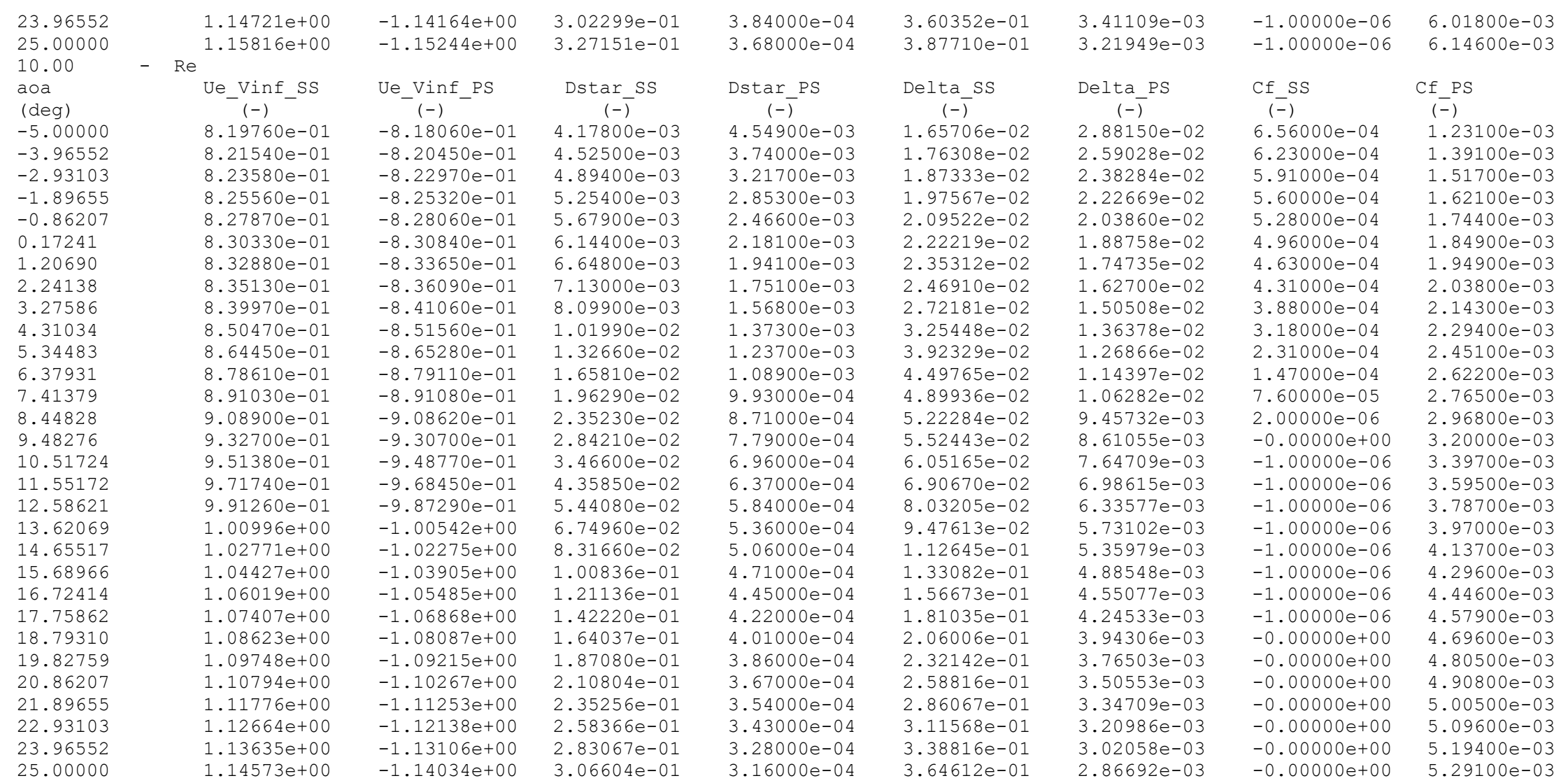




\section{A.3 Observer Positions}

The number and position of observers is set in the file ObserverLocations, which is explained in Appendix A.1. The positions must be specified in the OpenFAST global inertial frame coordinate system, which is located at the tower base and has the $\mathrm{x}$-axis pointing downwind, the y-axis pointing laterally, and the z-axis pointing vertically upward. A scheme of the coordinate system for the observers is shown in Figure A-1.

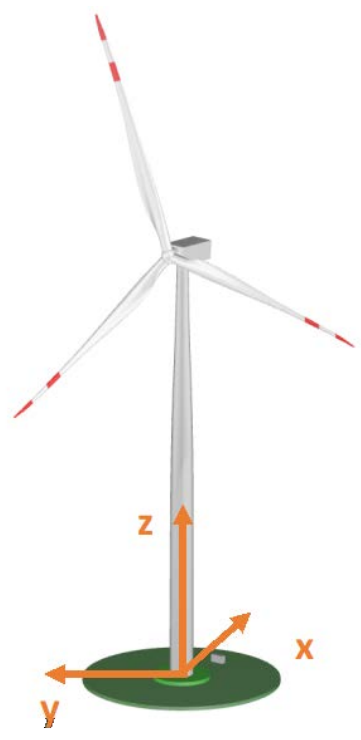

Figure A-1. Reference system for the observers

The International Energy Agency Wind Task 37 land-based reference wind turbine, which is shown in Table 1, has a hub height of 110 meters and a rotor radius of 65 meters, and has the International Electrotechnical Commission 61400-11 standards compliant observer located at:

$\mathrm{x}=175[\mathrm{~m}]$

$\mathrm{y}=0[\mathrm{~m}]$

$\mathrm{z}=0[\mathrm{~m}]$

An example of a file listing four observers located at a 2-meter height is shown here:

4 NrobsLoc - Total Number of observer locations

$\mathrm{X}$ Observer location in tower-base coordinate X horizontal (m), Y Observer location in tower-base coordinate Y Lateral (m), Z Observer location in tower-base coordinate Z

Vertical (m)

$-200 \quad-200 \quad 2$

$-200+200 \quad 2$

$+200-200 \quad 2$

$+200+200 \quad 2$ 


\section{A.4 Turbulence Grid}

When the flag TICalcMeth is set equal to 1 , the grid of incident turbulent intensity $I_{1}$ must be defined by the user. This is done by creating a file called TIGrid_In.txt, which mimics a TurbSim output file and contains a grid of turbulence intensity, which is defined as a fraction value. The file defines a grid centered at hub height and oriented with the OpenFAST global inertial frame coordinate system; see Figure A.1. A user-defined number of lateral and vertical points equally spaced by a user-defined number of meters must be specified. An example file for a 160 (lateral) by 180 (vertical) meters grid looks like the following:

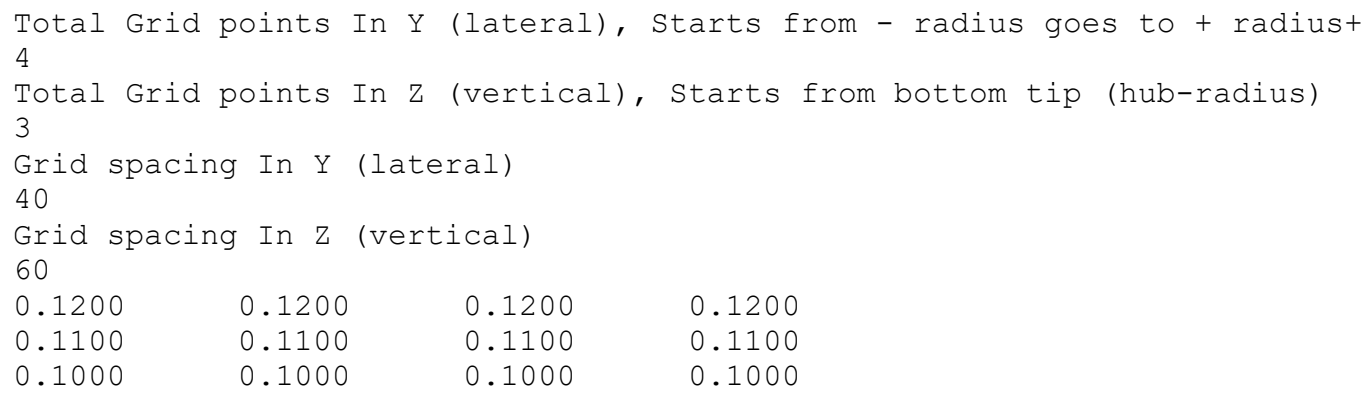

\section{A.5 Trailing-Edge Geometry}

When the flag BluntMod is set to 1, the detailed geometry of the trailing edge must be defined along the span. Two inputs must be provided, namely the angle, $\Psi$, between the suction and pressure sides of the profile, right before the trailing-edge point, and the height, $h$, of the trailing edge. $\Psi$ must be defined in degrees, while $h$ is in meters. Note that the BPM trailing-edge bluntness model is very sensitive to these two parameters, which, however, are often not easy to determine for real blades. Figure A-2 shows the two inputs.

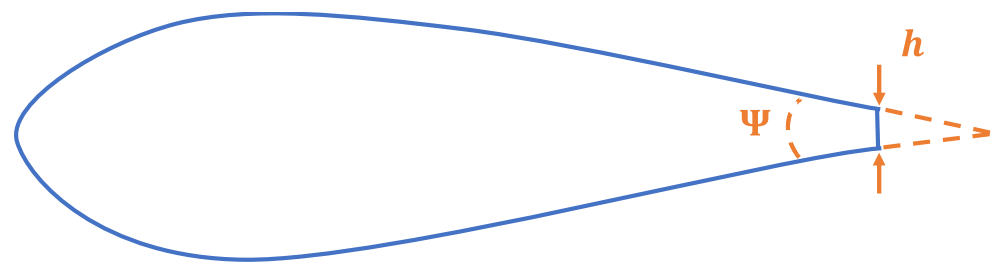

Figure A-2. Geometric parameters $\Psi$ and $h$ of the trailing-edge bluntness

The two distributions must be defined with the same spanwise resolution of the AeroDyn15 blade file, such as:

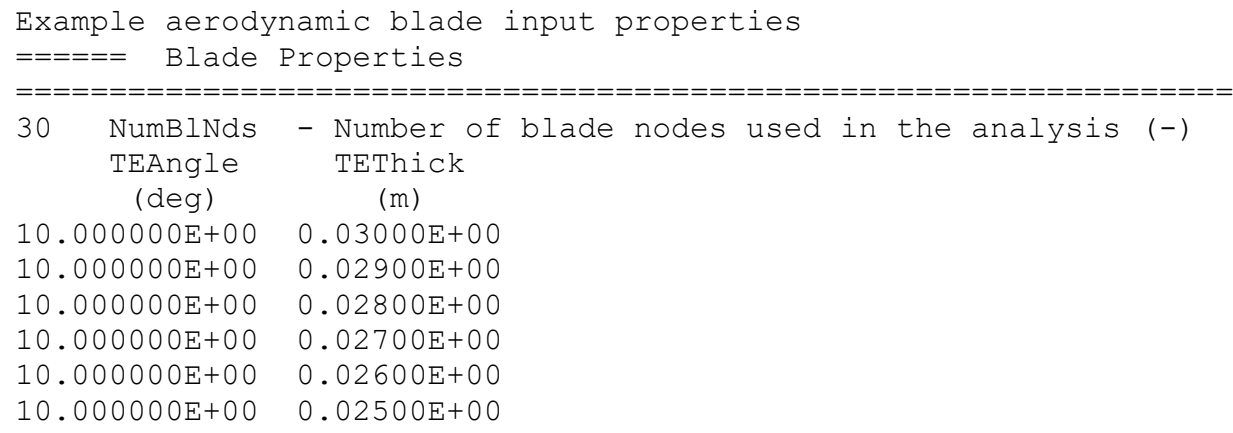




$\begin{array}{ll}10.000000 \mathrm{E}+00 & 0.02400 \mathrm{E}+00 \\ 10.000000 \mathrm{E}+00 & 0.02300 \mathrm{E}+00 \\ 10.000000 \mathrm{E}+00 & 0.02200 \mathrm{E}+00 \\ 10.000000 \mathrm{E}+00 & 0.02100 \mathrm{E}+00 \\ 10.000000 \mathrm{E}+00 & 0.02000 \mathrm{E}+00 \\ 10.000000 \mathrm{E}+00 & 0.01900 \mathrm{E}+00 \\ 10.000000 \mathrm{E}+00 & 0.01800 \mathrm{E}+00 \\ 10.000000 \mathrm{E}+00 & 0.01700 \mathrm{E}+00 \\ 10.000000 \mathrm{E}+00 & 0.01600 \mathrm{E}+00 \\ 10.000000 \mathrm{E}+00 & 0.01500 \mathrm{E}+00 \\ 10.000000 \mathrm{E}+00 & 0.01400 \mathrm{E}+00 \\ 10.000000 \mathrm{E}+00 & 0.01300 \mathrm{E}+00 \\ 10.000000 \mathrm{E}+00 & 0.01200 \mathrm{E}+00 \\ 10.000000 \mathrm{E}+00 & 0.01100 \mathrm{E}+00 \\ 10.000000 \mathrm{E}+00 & 0.01000 \mathrm{E}+00 \\ 10.000000 \mathrm{E}+00 & 0.01000 \mathrm{E}+00 \\ 10.000000 \mathrm{E}+00 & 0.01000 \mathrm{E}+00 \\ 10.000000 \mathrm{E}+00 & 0.01000 \mathrm{E}+00 \\ 10.000000 \mathrm{E}+00 & 0.01000 \mathrm{E}+00 \\ 10.000000 \mathrm{E}+00 & 0.01000 \mathrm{E}+00 \\ 10.000000 \mathrm{E}+00 & 0.01000 \mathrm{E}+00 \\ 10.000000 \mathrm{E}+00 & 0.01000 \mathrm{E}+00 \\ 10.000000 \mathrm{E}+00 & 0.01000 \mathrm{E}+00 \\ 10.000000 \mathrm{E}+00 & 0.01000 \mathrm{E}+00\end{array}$

33

This report is available at no cost from the National Renewable Energy Laboratory at www.nrel.gov/publications. 